\title{
Mechanism Design for Single Leader Stackelberg Problems and Application to Procurement Auction Design
}

\author{
Dinesh Garg and Y. Narahari, Fellow, IEEE
}

\begin{abstract}
In this paper, we focus on mechanism design for single leader Stackelberg problems, which are a special case of hierarchical decision making problems in which a distinguished agent, known as the leader, makes the first move and this action is followed by the actions of the remaining agents, which are known as the followers. These problems are also known as single leader rest follower (SLRF) problems. There are many examples of such problems in the areas of electronic commerce, supply chain management, manufacturing systems, distributed computing, transportation networks, and multiagent systems. The game induced among the agents for these problems is a Bayesian Stackelberg game, which is more general than a Bayesian game. For this reason, classical mechanism design, which is based on Bayesian games, cannot be applied as is for solving SLRF mechanism design problems. In this paper, we extend classical mechanism design theory to the specific setting of SLRF problems. As a significant application of the theory developed, we explore two examples from the domain of electronic commerce-first-price and second-price electronic procurement auctions with reserve prices. Using an SLRF model for these auctions, we derive certain key results using the SLRF mechanism design framework developed in this paper. The theory developed has many promising applications in modeling and solving emerging game theoretic problems in engineering.
\end{abstract}

Note to Practitioners-Hierarchical decision making problems arise naturally in many fields including electronic commerce, supply chain management, distributed computing, transportation networks, manufacturing systems, and multiagent systems. These problems involve interacting decision-making agents with a predefined sequence according to which decisions are taken. If the agents are rational and intelligent, these problems induce a Stackelberg game and for this reason, these problems could be called Stackelberg problems. In this paper, we look at a prominent special class of these problems called SLRF problems. There are many emerging examples of SLRF problems: auctions with reserve prices, Internet routing, supply chain formation, resource allocation in computational grids, routing in transportation networks, scheduling in manufacturing systems, etc. The main contribution of this paper is to extend classical mechanism design to the class of SLRF problems. Classical mechanism design uses the framework of Bayesian games whereas mechanism design applied to SLRF problems has to deal with the more general Bayesian Stackelberg games. This motivates the theoretical contribution of this paper, which is to extend mechanism design to

Manuscript received November 20, 2006; revised May 20, 2007. This paper was recommended for publication by Associate Editor M. Fanti and Editor P. Luh upon evaluation of the reviewers' comments. This work was supported in part by the Office of Naval Research (Code 313), Arlington, VA, under a Research Grant on Algorithmic Mechanism Design for Complex Game Theoretic Problems.

D. Garg is with IBM India Research Lab, Bangalore 560 071, India (e-mail: dingarg2@in.ibm.com).

Y. Narahari is with the Department of Computer Science and Automation, Indian Institute of Science, Bangalore 560 012, India (e-mail: hari@csa.iisc. ernet.in).

Digital Object Identifier 10.1109/TASE.2007.912717 the setting of SLRF problems. This paper also offers a significant application: designing incentive compatible procurement auctions with reserve prices. Mechanism design or protocol design in the context of all the emerging applications mentioned above will be a rich avenue for applying this theory.

Index Terms-Bayesian games, Bayesian Stackelberg games, incentive compatibility, mechanism design, procurement auctions with reserve prices, single leader rest follower (SLRF) games, Stackelberg equilibrium.

\section{ACRONYMS}

SCF Social choice function.

SLRF Single leader rest followers.

BaSIC Bayesian Stackelberg incentive compatible.

PAR Procurement auction with reserve price.

F-PAR First-price procurement auction with reserve price.

S-PAR Second-price procurement auction with reserve price.

\section{INTRODUCTION}

$\mathbf{H}$ IERARCHICAL decision making problems arise naturally in many fields including Economics, Sociology, Engineering, Operations Research, Control Theory, Computer Science, Electronic Commerce, Supply Chain Management, Transportation Networks, and Manufacturing Systems. These problems involve rational and intelligent decision-making individuals or agents who interact, following a hierarchical structure. In these problems, there is a predefined sequence based on the hierarchy and the decision makers need to take their decision (or action) according to that particular sequence. The decision makers have their independent and perhaps conflicting objectives which they try to maximize independently of the rival decision makers, but their objectives are affected by the actions of the rivals as an externality. The game induced among the decision makers for such a problem was first studied by Stackelberg [1] and from then onward these games are popularly known as Stackelberg games.

A prominent category of hierarchical decision-making problems is the set of problems where the first move is made by a subset of decision makers by taking their action in a simultaneous manner and the next move is made by the rest of the decision makers by taking their action in a simultaneous manner. The players who make the first move are called the leaders and 
the players who make the second move are called the followers. In such problems, the actions of the leaders can influence the actions of the followers. A further subclass of these problems is the set of problems where there is only one leader and rest of the decision makers are followers. These problems are known as single leader Stackelberg problems or single leader rest follower (SLRF) problems.

\section{A. Examples of SLRF Problems}

We first succinctly describe several emerging examples of SLRF problems in the real-world.

Task Allocation in Distributed Systems: Consider a distributed system with a centralized scheduler which allocates jobs to individual processors in a distributed/parallel/grid computing environment. The scheduling policy adopted by the centralized scheduler affects the scheduling strategies to be adopted by the local schedulers of individual machines.

Internet Routing: The access networks situated at the edge of the Internet are connected to the rest of the Internet through a tiered hierarchy of Internet Service Providers (ISPs). At the top of this hierarchy is a relatively small number of so called Tier-1 ISPs or Internet Backbone such as UUNet, Sprint, AT\&T, Genuity, and Cable and Wireless. A Tier-2 ISP typically has regional or national coverage and, thus, in order to reach a large portion of the global Internet, it needs to route traffic through one of the Tier-1 ISPs to which it is connected. Access ISPs (for example, residential ISPs such as $A O L$, and company ISPs) are at the bottom of the hierarchy and access the entire Internet by routing the traffic through a Tier-2 ISP [2]. In such a hierarchical scheme, the routing policy and tariff plan of a Tier-1 ISP affects the routing decisions and bandwidth selling price of corresponding Tier-2 ISPs.

Admission, Routing, and Scheduling in Networks: In any wide area network, there is a well-defined hierarchy of decision layers in terms of admission control (highest layer), routing policies (second layer), and scheduling policies (lowest layer). In a large-scale factory network, we have a similar hierarchy in terms of input control or part release policies (highest layer), routing to individual factory cells (second layer), and scheduling of jobs within individual cells.

Routing in Transportation Networks: The policies of a State transportation authority for streamlining traffic flows in a metro affect the routing decisions of end users. Mechanism design is required to control congestion in such networks; here, the transportation authority is the leader and end users are the followers.

Resource Allocation in Governance: The energy policies, water resource policies, and budget planning of a Central/Federal Government affect the objectives and options, and hence the strategies, of individual state governments. This process continues through a hierarchy of decision-makers, including local governments, planning agencies, and basic economic units such as firms and households.

Electricity Distribution by a National Power Grid: The policy set forth by the Central Electricity Regulatory Commission regarding tariff and distribution of the electricity among various states, affects the tariff and subsidy plans of the state governments. This eventually affects the consumption patterns of basic economic units such as industries and households.
Electronic Commerce: In electronic commerce, SLRF problems arise in both forward auctions (for selling items) and reverse auctions (for procuring items). In a forward auction, the reservation prices announced by the seller will affect the bidding patterns of the buying agents. In procurement auctions, announcement by the buyer of a maximum willingness to pay will affect the bidding patterns of the sellers. In fact, procurement auctions with reserve prices constitute a primary motivation for this paper. We discuss this example next.

\section{B. Motivating Example: Procurement Auction With Reserve Price (PAR)}

Consider an electronic procurement marketplace where a buyer $b$ registers herself and wishes to procure a single indivisible object. Let us assume that there are $n$ potential sellers, indexed by $i=1,2, \ldots, n$, who also register themselves with the marketplace. The marketplace first invites the buyer to report her type. Here by type of an individual, we mean the worth he or she attaches to the object. Based on her actual type $\theta_{b}$, the buyer reports her type to be, say $\hat{\theta}_{b}$, to the marketplace. The declared type of the buyer, that is $\hat{\theta}_{b}$, is treated as the price above which the buyer is not willing to buy the object. This price is known as reserve price. The marketplace publicly announces this reserve price among all the sellers. Now, the sellers are invited to report their types confidentially to the marketplace. Based on actual type $\theta_{i}$, each seller $i$ reports his type, say $\hat{\theta}_{i}$, to the marketplace. The reported type of the seller $i$, that is $\hat{\theta}_{i}$ is treated as the price below which the seller $i$ is not willing to sell the object. After receiving the types from all the sellers, the marketplace determines the winning seller, the amount that will be paid to him, and the amount that will be paid by the buyer. These are called as winner determination and payment rules. In practice, such a trading institution is known as procurement auction with reserve prices (PAR). Depending on which winner determination and payment rules are employed by the marketplace, it may take different forms. Following are two interesting instances.

1) First-Price Procurement Auction With Reserve Prices (F-PAR): In this setting, the marketplace first discards all those sellers whose reported types fall above the reserve price announced by the buyer. Next, the seller whose reported type is the lowest among the remaining sellers is declared as the winner. The winner transfers the object to the buyer and the buyer pays to the winning seller an amount equal to his reported type, that is $\hat{\theta}_{i}$. If there is no type reported below the reserve price, then no deal is struck. On the other hand, if there is a tie among the winning sellers then the winner is chosen randomly, where each of the lowest valued types has an equal chance of winning.

2) Second-Price Procurement Auction With Reserve Prices $(S-P A R)$ : In this setting, the marketplace first discards all those sellers whose reported types fall above the reserve price. Next, the seller whose type is the lowest among the remaining sellers is declared the winner. The winning seller transfers the object to the buyer and the buyer pays to him an amount equal to the second lowest type, if such a type exists, otherwise an amount equal to the reserve price. Further, if there is no type reported below the reserve price then no deal is struck. If there is a tie 
among winning sellers, the winner is chosen randomly, where each of the lowest type has an equal chance of winning. ${ }^{1}$

The game theoretic model that is appropriate for situations of the above kind are the single leader rest follower (SLRF) games. SLRF games are, in turn, a special case of the so called Stackelberg games [1].

\section{Relevant Work}

Classical mechanism design theory [3] uses the framework of Bayesian games, which are noncooperative games with incomplete information [4]. Mechanism design applied to SLRF problems has to deal with Bayesian Stackelberg games, which are more general than Stackelberg games. To the best of our knowledge, there is as yet no comprehensive treatment of mechanism design as applied to Stackelberg problems in general and SLRF problems, in particular, this provides the primary motivation for this paper. We first briefly review the work in the literature which comes closest to the topic we are addressing here.

The paper by Cremer and Riordan [5] considers the classical public goods problem where there is a finite set of agents $\{1,2, \ldots, n\}$ and a mechanism is required for allocating resources among the agents based on the preferences announced by the agents. For this problem, the paper considers a class of sequential mechanisms where (i) the first agent (agent 1) plays before the others, with only incomplete information about the types of the rest of the players; and (ii) all other agents (agents 2 through $n$ ) play subsequently, in any arbitrary order, and have a dominant strategy. The authors further restrict their attention to the class of such sequential mechanisms in which (i) truth telling is a dominant strategy for all the agents except the first agent (i), the truth telling is an equilibrium strategy for all the agents. The authors call such a mechanism as a Stackelberg mechanism. The authors derive a sufficiency condition under which a Stackelberg mechanism is both allocatively efficient (that is, the allocation maximizes the total value of allocated agents) and budget balanced (that is, the total receipts is equal to the total payments). The class of mechanisms considered by Cremer and Riordan is a very special class of mechanisms treated by us in the current paper. This will be explained soon in the next section.

In [6], Chang and Luh consider two person, single-stage, deterministic Stackelberg games with incomplete information, and come up with a necessary and sufficient condition for the existence of an optimal strategy for the leader. In the event that an optimal strategy does not exist, they show that an $\epsilon$-optimal strategy always exists provided that the leader's cost is bounded. These results are extended to three-level Stackelberg decision problems by Luh et al. [7] and to multistage Stackelberg games by Luh et al.[8]. These contributions are from a control theoretic perspective and not from a mechanism design perspective. For example, these papers implicitly assume incentive compatibility on the part of all the players of the game.

Konrad and Leininger [9] consider a set of $n$ players who are arbitrarily partitioned into a group of players who choose their efforts early and a group of players who choose late. Only the player with the lowest cost of effort has a positive payoff

\footnotetext{
${ }^{1}$ If there are exactly two reported types below the reserve price and they are equal then any one of them is declared as the winner at random and the amount that the buyer pays to the winner is equal to this lowest type.
}

in any equilibrium. It is shown that the strongest player typically chooses late, whereas all other players are indifferent with respect to their choice of timing. In the most prominent equilibrium, the player with the lowest cost of effort wins the auction at zero aggregate cost. This paper discusses design of a very specific type of auction in a Stackelberg setting and does not consider the general setting of single leader Stackelberg problems.

\section{Contributions of This Paper}

The existing literature on mechanism design in the context of Stackelberg problems addresses only special classes of problems in specific problem settings. Also, classical mechanism design cannot be directly applied as is for Stackelberg mechanism design problems. Thus, there is an important need to develop a general mechanism design framework for Stackelberg mechanism design problems. As a significant first step in this direction, we consider in this paper a major special class of Stackelberg problems, namely, single leader rest follower (SLRF) problems, and extend classical mechanism design theory to the class of SLRF problems. The following are our specific contributions. 1) First, we formally define the mechanism design problem as it applies to the context of SLRF mechanism design problems. We develop a theory of mechanism design for SLRF problems on the lines of classical mechanism design theory by focusing on the following aspects.

- SLRF social choice functions (SCFs).

- SLRF indirect mechanisms.

- SLRF direct revelation mechanisms.

- Implementing an SLRF SCF in Bayesian Stackelberg equilibrium.

- Bayesian Stackelberg incentive compatibility (BaSIC) of an SLRF SCF.

- The revelation principle for Bayesian Stackelberg equilibrium.

We state and prove the following two results which are key to SLRF mechanism design.

- Proposition 3.1 and Corollary 3.1 show that if a mechanism implements a social choice function in dominant strategy equilibrium, then it also implements the social choice function in Bayesian Stackelberg equilibrium.

- Proposition 3.2 asserts that a social choice function that is dominant strategy incentive compatible is also BaSIC.

Note that we address SLRF mechanism design problems in all their generality, while Cremer and Riordan [5] address a very special class of SLRF problems, where the mechanism is BaSIC for the leader as well as the followers and, moreover, the mechanism is dominant strategy incentive compatible for the followers. 2) We explore two examples as a significant application and validation of the theory developed. These are: first-price procurement auction with reserve prices (F-PAR) and second-price procurement auction with reserve prices (S-PAR). We model these as SLRF problems and using the mechanism design framework for SLRF problems, we investigate the Bayesian Stackelberg incentive compatibility of these two SLRF mechanisms and derive two important results. In particular, we show that under some mild conditions, F-PAR is BaSIC neither for the buyer nor for the sellers, whereas S-PAR is BaSIC for the sellers but not for the buyer. The above results 
for F-PAR and S-PAR can be deduced from similar results available for forward auctions in the literature, however, the approach that we have used to derive the results (based on the theory developed in this paper) is quite novel and different.

To set the stage for the main contributions of this paper, we first present, in Section II, a succinct overview of the Stackelberg games and SLRF games. The material in Section II is adapted from Basar [10] to the specific needs of this paper. Section III is devoted to extending mechanism design to the specific setting of SLRF problems. Section IV describes the application of the theory to F-PAR and S-PAR mechanisms. In Section V, we conclude this paper by suggesting several promising directions for future work.

\section{STACKELBERG GAMES AND SLRF GAMES}

Noncooperative games with an additional structure of hierarchical decision making were first studied by Stackelberg [1]. Games with a hierarchical decision making structure are known as Stackelberg games and the solution concept used for these games is the Stackelberg equilibrium. A comprehensive treatment of the Stackelberg games can be found in [10].

To begin with, we consider the following noncooperative $f$ nite game with complete information in strategic form:

$$
\Gamma=\left(N,\left(S_{i}\right)_{i \in N},\left(u_{i}\right)_{i \in N}\right)
$$

where $N$ is a nonempty set of the $n$ players (or agents), and, for each $i$ in $N, S_{i}$ is a nonempty set of strategies (or pure strategies) available to player $i$, and $u_{i}$ is a function from $S$ into the set of real numbers $\mathbb{R}$, where $S=S_{1} \times \cdots \times S_{n}=\times_{j \in N} S_{j}$. If we let $s=\left(s_{1}, \ldots, s_{n}\right)$ denote the set of strategies chosen by all the $n$ players, then we call $s$ as a strategy profile of the agents. Thus, we can see that set $S$ denotes the set of all possible strategy profiles and for any strategy profile $s \in S$, the number $u_{i}(s)$ represents the expected utility payoff that player $i$ would get.

In the above description of the game $\Gamma$, it is an implicit assumption that all the players choose their strategies simultaneously. It is possible to impose an additional structure of hierarchical decision making on this game where agents choose their strategies in a sequential manner as suggested by the hierarchy. The hierarchy is defined as a sequence of subsets of players, $H_{1}, H_{2}, \ldots, H_{h}$ which satisfies the following feasibility conditions:

$$
\begin{aligned}
h & \leq|N| \\
H_{j} & \subseteq N \quad \forall j=1, \ldots, h \\
H_{j} & \neq \emptyset \quad \forall j=1, \ldots, h \\
H_{i} \cap H_{j} & =\emptyset \quad \forall i \neq j
\end{aligned}
$$

where $h$ represents the total number of levels in the hierarchy. Given a hierarchy $H$, the players now choose their strategies in the following manner. First, all the players at hierarchy level 1, i.e., $H_{1}$, choose their strategies simultaneously. The strategies chosen by these players are announced publicly to the rest of the players. Next, all the players at hierarchy level 2, i.e., $\mathrm{H}_{2}$, choose their strategies simultaneously and again the chosen strategies by all these players are announced publicly to the rest of the players. This process continues until all the players choose their strategies. At the end of the process, the utility of each player $i$ is computed by using the function $u_{i}(s)$, where $s=\left(s_{i}\right)_{i \in N}$ is profile of strategies chosen by all the players in a hierarchical manner. Note that under such a hierarchical scheme, when the players at a given level of hierarchy $H_{j}$ are about to choose their strategies, the strategies chosen by the players in hierarchy levels $j$ through $h-1$ is a common knowledge among them.

A strategic form game $\Gamma$ superimposed with a hierarchy of decision making is called as Stackelberg game and can be represented in the following fashion ${ }^{2}$

$$
\Gamma_{s}=\left(\left(H_{j}\right)_{j=1, \ldots, h},\left(S_{i}\right)_{i \in N},\left(u_{i}\right)_{i \in N}\right) .
$$

Following are two special cases of the Stackelberg games that are of interest to us.

Simultaneous Move Games ( $h=1)$ : In this case, there is only one level of hierarchy and the Stackelberg game $\Gamma_{s}$ just boils down to the noncooperative game $\Gamma$. For such games, one can use the standard solution concepts available in the literature of noncooperative game theory, for example, dominant strategy equilibrium and Nash equilibrium.

Leader-Follower Games $(h=2)$ : In this case, the players are divided into two levels of hierarchy $-H_{1}$ and $H_{2}$. The players in $H_{1}$ are called leaders because they are the ones who choose their strategies first. The players in $H_{2}$ are called followers and all of them, after observing the strategies chosen by the leaders, choose their strategies in a simultaneous manner. Within the class of leader-follower games, there is an interesting subclass of games where $H_{1}$ is a singleton. Such games are called as SLRF games. In the remainder of this paper, we will be just focusing on SLRF games.

\section{A. Pure Strategy Stackelberg Equilibrium for SLRF Games}

The pure strategy Stackelberg equilibrium of the SLRF games is based on following two quantities.

1) The set of followers' optimal response strategy profiles.

2) The set of leader's secure strategies.

Now, we define each of these two quantities one by one.

1) The Set of Followers' Optimal Response Strategy Profiles: Let us assume that the leader has chosen her strategy to be $s_{l} \in S_{l}$. The following set of pure strategy profiles of the followers, denoted by $R\left(s_{l}\right)$, is called as the set of followers' optimal response (or rational reaction) strategy profiles

$$
\begin{aligned}
R\left(s_{l}\right)=\left\{s_{-l} \in S_{-l} \mid u_{j}\right. & \left(s_{l}, s_{j}, s_{-l, j}\right) \\
& \left.\leq u_{j}\left(s_{l}, s_{-l}\right) \forall s_{j} \in S_{j} \forall j \in N \backslash\{l\}\right\}
\end{aligned}
$$

where

- $S_{-l}=\times_{j \in N \backslash\{l\}} S_{j}$.

- $\left(s_{l}, s_{-l}\right)$ is a strategy profile of the players in which the leader plays with strategy $s_{l}$ and the followers play with strategy profile $s_{-l}$.

- $\left(s_{l}, s_{j}, s_{-l, j}\right)$ is a strategy profile in which the leader plays with strategy $s_{l}$, the follower $j$ plays with strategy $s_{j}$, and rest of the followers play with the same strategy as suggested by $s_{-l}$.

${ }^{2}$ The games with a hierarchy of decision making have also been studied in extensive form but this is not required for our discussion here. For more details on extensive form Stackelberg games, see [10]. 
The above definition essentially says that for any fixed pure strategy $s_{l}$ of the leader, the rational reaction set $R\left(s_{l}\right)$ consists of all the pure strategy Nash equilibria of the following $(N-1)$ players game $\Gamma_{-l}$ :

$$
\Gamma_{-l}=\left((N \backslash\{l\}),\left(S_{i}\right)_{i \in N \backslash\{l\}},\left(\bar{u}_{i}\right)_{i \in N \backslash\{l\}}\right)
$$

where $\bar{u}_{i}\left(s_{-l}\right)=u_{i}\left(s_{l}, s_{-l}\right) \quad \forall s_{-l} \in S_{-l}, \quad \forall i \in(N \backslash\{l\})$. It is easy to see that nothing prevents $R\left(s_{l}\right)$ from being an empty set.

2) The Set of Leader's Secure Strategies: Assuming that $R\left(s_{l}\right)$ is a nonempty set for each $s_{l} \in S_{l}$, a strategy $s_{l}^{*} \in S_{l}$ of the leader $l$ is said to be a secure strategy if it satisfies the following security constraint:

$$
s_{l}^{*} \in \arg \max _{s_{l} \in S_{l} s_{-l} \in R\left(s_{l}\right)} u_{l}\left(s_{l}, s_{-l}\right) .
$$

Note that under the assumption that the set $R\left(s_{l}\right)$ is nonempty for each $s_{l} \in S_{l}$, a secure strategy $s_{l}^{*}$ always exists if the set $S_{l}$ and the set $R\left(s_{l}\right)$ are both finite. A secure strategy for the leader maximizes the leader's utility against the worst possible play by the followers. For this reason, the leader will always like to play a secure strategy.

3) Stackelberg Equilibrium: A strategy profile $s^{*}=$ $\left(s_{l}^{*}, s_{-l}^{*}\right)$ is said to be a Stackelberg equilibrium if $s_{l}^{*}$ is a secure strategy for the leader and $s_{-l}^{*}$ is an optimal response strategy of the followers against $s_{l}^{*}$, that is $s_{-l}^{*} \in R\left(s_{l}^{*}\right)$.

\section{B. Bayesian SLRF Games}

Here, we impose a hierarchical decision making structure on Bayesian games, which are basically finite noncooperative games with incomplete information, and study the solution concept for the resulting games. A comprehensive treatment of the Bayesian games can be found in [4].

To begin with, we consider the following noncooperative finite game with incomplete information (also known as Bayesian game):

$$
\Gamma^{b}=\left(N,\left(C_{i}\right)_{i \in N},\left(\Theta_{i}\right)_{i \in N},\left(\phi_{i}\right)_{i \in N},\left(u_{i}\right)_{i \in N}\right)
$$

where $N$ is a nonempty set of $n$ players, and, for each $i \in$ $N, C_{i}$ is a nonempty set of actions ${ }^{3}$ available to player $i, \Theta_{i}$ is a nonempty set of possible types of player $i, \phi_{i}: \Theta_{i} \rightarrow \Delta \Theta_{-i}$ is a belief function which gives the subjective probability of player $i$ about the types of the other players for a given type of his own, and $u_{i}: C \times \Theta \rightarrow \mathbb{R}$ is the utility function of player $i$, where $C=C_{1} \times \cdots \times C_{n}$, and $\Theta=\Theta_{1} \times \ldots \times \Theta_{n}$. A pure strategy $s_{i}$ for player $i$ in the Bayesian game $\Gamma^{b}$ is defined as a function from $\Theta_{i}$ to $C_{i}$. Also, it is easy to see that $s_{i} \in \mathrm{\times}_{\Theta_{i}} C_{i}$. The set $C_{i}$ represents the set of pure strategies for player $i$. We denote the set of pure strategies of player $i$ as $S_{i}$, that is $S_{i}=\times_{\Theta_{i}} C_{i}$. We say that the game $\Gamma^{b}$ is finite iff the sets $N, C_{i}$, and $\Theta_{i}$ (for

\footnotetext{
${ }^{3}$ Note that we call these elements actions instead of pure strategies. The pure
} strategies will be defined shortly. every $i$ ) are all finite. Throughout this paper, we confine our discussion to finite Bayesian games unless otherwise stated.

In the above description of the Bayesian game $\Gamma^{b}$, it is an implicit assumption that after learning their types, all the players choose their actions simultaneously. However, in a way similar to the complete information case, it is possible to impose an additional structure of hierarchical decision-making, where players after learning their types, choose their actions in a sequential manner as suggested by the hierarchy $H=H_{1}, H_{2}, \ldots, H_{h}$, where $H$ satisfies the properties (1)-(4). A Bayesian game $\Gamma^{b}$ together with hierarchical decision making can be called Bayesian Stackelberg game and will have the following components:

$$
\Gamma_{s}^{b}=\left(\left(H_{j}\right)_{j=1, \ldots, h},\left(C_{i}\right)_{i \in N},\left(\Theta_{i}\right)_{i \in N},\left(\phi_{i}\right)_{i \in N},\left(u_{i}\right)_{i \in N}\right) .
$$

It is now apparent that a SLRF Bayesian Stackelberg game is the one in which one player is declared as the leader and after learning her type, she first takes her action. The action taken by the leader becomes common knowledge among the followers but her type remains unknown to the followers. Following the action of the leader, all the followers, who have already learned their types, take their actions simultaneously. In what follows, we characterize the Bayesian Stackelberg equilibrium solution concept for such games. ${ }^{4}$

\section{Pure Strategy Bayesian Stackelberg Equilibrium for Bayesian SLRF Games}

1) The Set of Followers' Optimal Response Strategy Profiles: Let us assume that after learning her type $\theta_{l} \in \Theta_{l}$, the leader takes an action $c_{l} \in C_{l}$. For any such action $c_{l}$, the set $R\left(c_{l}\right)$ below, which is a set of pure strategy profiles of the followers, is called as the set of followers' optimal response (or rational reaction) strategy profiles:

$$
\begin{aligned}
R\left(c_{l}\right)= & \left\{s_{-l} \in S_{-l} \mid v_{\theta_{j}}\left(c_{l}, c_{j}, s_{-l, j}\right)\right. \\
& \leq v_{\theta_{j}}\left(c_{l}, s_{-l}\right), \quad \forall c_{j} \in C_{j}, \\
& \left.\forall \theta_{j} \in \Theta_{j}, \quad \forall \quad j \in N \backslash\{l\}\right\}
\end{aligned}
$$

where

- $S_{-l}=\times_{j \in N \backslash\{l\}} S_{j}$.

- $\left(c_{l}, s_{-l}\right)$ is an action-strategy profile of the players in which the leader takes an action $c_{l}$ and the followers take actions as suggested by the corresponding pure strategy for them in the profile $s_{-l}$.

- $\left(c_{l}, c_{j}, s_{-l, j}\right)$ is an action-strategy profile in which the leader takes an action $c_{l}$, the follower $j \in N$ takes an action $c_{j}$ and rest of the followers take actions as suggested by the corresponding pure strategy for them in the profile $s_{-l}$.

- The quantities $v_{\theta_{j}}\left(c_{l}, s_{-l}\right)$ and $v_{\theta_{j}}\left(c_{l}, c_{j}, s_{-l, j}\right)$ are the expected payoffs, respectively, to the player $j$ when his type

${ }^{4}$ In the rest of the discussion, by SLRF game we mean SLRF Bayesian Stackelberg game unless otherwise stated. 
is $\theta_{j}$, and the players follow the action-strategy profile $\left(c_{l}, s_{-l}\right)$ and $\left(c_{l}, c_{j}, s_{-l, j}\right)$, respectively. The following are the precise formulae for $v_{\theta_{j}}(\cdot)$ :

$$
\begin{aligned}
& v_{\theta_{j}}\left(c_{l}, s_{-l}\right)= \\
& \quad \sum_{\theta_{-j} \in \Theta_{-j}} \phi_{j}\left(\theta_{-j} \mid \theta_{j}\right) u_{j}\left(\left(c_{l}, s_{-l}\left(\theta_{-l}\right)\right),\left(\theta_{j}, \theta_{-j}\right)\right) \\
& v_{\theta_{j}}\left(c_{l}, c_{j}, s_{-l, j}\right)= \\
& \quad \sum_{\theta_{-j} \in \Theta_{-j}} \phi_{j}\left(\theta_{-j} \mid \theta_{j}\right) u_{j}\left(\left(c_{l}, c_{j}, s_{-l, j}\left(\theta_{-l, j}\right)\right),\left(\theta_{j}, \theta_{-j}\right)\right)
\end{aligned}
$$

where $\theta=\left(\theta_{l}, \theta_{-l}\right)=\left(\theta_{j}, \theta_{-j}\right)=\left(\theta_{l}, \theta_{j}, \theta_{-l, j}\right)$..

The above definition of $R\left(c_{l}\right)$ essentially says that for any action $c_{l}$ of the leader, the optimal response set $R\left(c_{l}\right)$ consists of all the pure strategy profiles of the followers that are pure strategy Bayesian Nash equilibria of the following $(N-1)$ player Bayesian game $\Gamma_{-1}^{b}$ :

$$
\begin{aligned}
\Gamma_{-l}^{b}= & \left(N \backslash\{l\},\left(C_{i}\right)_{i \in N \backslash\{l\}},\left(\Theta_{i}\right)_{i \in N \backslash\{l\}},\right. \\
& \left.\left(\psi_{i}\right)_{i \in N \backslash\{l\}},\left(\bar{u}_{i}\right)_{i \in N \backslash\{l\}}\right)
\end{aligned}
$$

where

$$
\begin{aligned}
& \psi_{i}: \Theta_{i} \rightarrow \Delta \Theta_{-l, i} \\
& \psi_{i}\left(\theta_{-l, i} \mid \theta_{i}\right)=\sum_{\theta_{l} \in \Theta_{l}} \phi_{i}\left(\left(\theta_{-l, i}, \theta_{l}\right) \mid \theta_{i}\right) \\
& \bar{u}_{i}\left(c_{-l}, \theta_{-l}\right)=\sum_{\theta_{l} \in \Theta_{l}} \phi_{i}\left(\theta_{l} \mid\left(\theta_{-l, i}, \theta_{i}\right)\right) u_{i} \\
& \times\left(\left(c_{l}, c_{-l}\right),\left(\theta_{l}, \theta_{-l}\right)\right) .
\end{aligned}
$$

It is easy to see that $R\left(c_{1}\right)$ may be an empty set as well. However, $R\left(c_{l}\right)$ is otherwise always a finite set under the assumption that the game $\Gamma_{s}^{b}$ is a finite game, which means the sets $N,\left(C_{i}\right)_{i \in N}$, and $\left(\Theta_{i}\right)_{i \in N}$ are finite sets for each $i \in N$. If we allow the sets $\Theta_{i}$ to be infinite, then the relation (5) remains the same (except requiring the finiteness of the quantities $v_{\theta_{j}}\left(c_{l}, s_{-l}\right)$ and $v_{\theta_{j}}\left(c_{l}, c_{j}, s_{-l, j}\right)$ ) but the relations (6) and (7) get modified in the following manner:

$$
\begin{aligned}
& v_{\theta_{j}}\left(c_{l}, s_{-l}\right)=E_{\theta_{-j}}\left[u_{j}\left(\left(c_{l}, s_{-l}\left(\theta_{-l}\right)\right),\left(\theta_{j}, \theta_{-j}\right)\right) \mid \theta_{j}\right] \\
& v_{\theta_{j}}\left(c_{l}, c_{j}, s_{-l, j}\right)=E_{\theta_{-j}} \\
& \quad \times\left[u_{j}\left(\left(c_{l}, c_{j}, s_{-l, j}\left(\theta_{-l, j}\right)\right),\left(\theta_{j}, \theta_{-j}\right)\right) \mid \theta_{j}\right] .
\end{aligned}
$$

Now, it is also quite possible that the set $R\left(c_{l}\right)$ is empty.

2) Secure Strategy Set of Leader: Assuming that $R\left(c_{l}\right)$ is nonempty for each $c_{l} \in C_{l}$, we call a strategy $s_{l}^{*} \in S_{l}$ of the leader $l$ to be a secure strategy if it satisfies the following security constraint for each $\theta_{l} \in \Theta_{l}$

$$
\begin{array}{r}
s_{l}^{*}\left(\theta_{l}\right) \in \arg \max _{c_{l} \in C_{l}}\left[\min _{s_{-l} \in R\left(c_{l}\right)} \sum_{\theta_{-l} \in \Theta_{-l}} \phi_{l}\left(\theta_{-l} \mid \theta_{l}\right) u_{l}\right. \\
\left.\times\left(c_{l}, s_{-l}\left(\theta_{-l}\right),\left(\theta_{l}, \theta_{-l}\right)\right)\right] .
\end{array}
$$

A leader will always play a secure strategy. An implicit assumption behind the above relation is that the game $\Gamma_{s}^{b}$ is a finite game. However, if we allow the sets $\Theta_{i}$ to be infinite, then the relation (10) gets modified in the following manner:

$$
\begin{aligned}
s_{l}^{*}\left(\theta_{l}\right) \in \arg \max _{c_{l} \in C_{l}}\left[\min _{s_{-l}} \in R\left(c_{l}\right)\right. & E_{\theta_{-l}} \\
& \left.\times\left[u_{l}\left(c_{l}, s_{-l}\left(\theta_{-l}\right),\left(\theta_{l}, \theta_{-l}\right)\right) \mid \theta_{l}\right]\right] .
\end{aligned}
$$

Further, if we allow the sets $\left(C_{i}\right)_{i \in N}$ to be infinite, then the above relation will reduce to the following form:

$$
\begin{aligned}
s_{l}^{*}\left(\theta_{l}\right) \in \arg \sup _{c_{l} \in C_{l}}\left[\inf _{s_{-l}} \in R\left(c_{l}\right)\right. & E_{\theta_{-l}} \\
& \left.\times\left[u_{l}\left(c_{l}, s_{-l}\left(\theta_{-l}\right),\left(\theta_{l}, \theta_{-l}\right)\right) \mid \theta_{l}\right]\right] .
\end{aligned}
$$

3) Pure Strategy Bayesian Stackelberg Equilibrium: A strategy profile $s^{*}(\cdot)=\left(s_{l}^{*}(\cdot), t_{-l}^{*}(\cdot)\right)$ is said to be a Bayesian Stackelberg equilibrium if $s_{l}^{*}(\cdot)$ is a secure strategy for the leader and $t_{-l}^{*}: C_{l} \rightarrow \cup_{c_{l} \in C_{l}} R\left(c_{l}\right)$ is a rational reaction strategy of followers against $s_{l}^{*}(\cdot)$, that is $t_{-l}^{*}\left(s_{l}^{*}\left(\theta_{l}\right)\right) \in R\left(s_{l}^{*}\left(\theta_{l}\right)\right) \quad \forall \theta_{l} \in \Theta_{l}$.

\section{Mechanism Design fOR SLRF Problems}

\section{A. SLRF Mechanism Design Problem}

The crucial difference between classical mechanism design and mechanism design for SLRF problems is the condition on the sequence in which the agents take their decisions. We assume that the mechanism is implemented by a social planner or policy maker or mediator. We use the phrase social planner henceforth. Recall that in an SLRF mechanism design problem, there is a special agent $l \in N$, the leader. The social planner first invites the leader to take an action after the leader has learned her type. The action taken by the leader is announced by the social planner among the rest of the agents, called the followers. However, the leader's type is still unknown to the followers as well as social planner. Next, the social planner invites all the followers, who have already learned their types, to choose their actions simultaneously.

Similar to the classical mechanism design problem, here also, the social planner faces the same problems of information elicitation and preference aggregation and, hence, the planner uses social choice function to aggregate the preferences of the leader and the followers into a single outcome $x \in X$. An outcome $x$ represents a particular way in which the given social choice problem can be resolved. For example, under the usual auction setting, an outcome would represent how the winners are chosen in the auction and the vector of payments to be made by the different agents. We first define an SLRF social choice function.

\section{B. SLRF Social Choice Function}

Definition 3.1: An SLRF social choice function (SCF) is a function $f: \Theta \rightarrow X$, which a social planner uses to assign a collective choice $f\left(\theta_{l}, \theta_{-l}\right)$ to each possible profile $\left(\theta_{l}, \theta_{-l}\right) \in$ $\Theta$ of the leader and the followers' types.

A social choice function aggregates and maps the preferences of the leader and the followers to an outcome. It associates an outcome to each profile of preferences of the leader and the followers. 


\section{SLRF Indirect Mechanism}

Definition 3.2: An SLRF mechanism M $\mathrm{M}_{\mathrm{SLRF}}=$ $\left(C_{l},\left(C_{i}\right)_{i \in N \backslash\{l\}}, g(\cdot)\right)$ is a collection of the action set of the leader $C_{l}$, action sets of followers $\left(C_{i}\right)_{i \in N \backslash\{l\}}$, and an outcome function $g: C \rightarrow X$, where $C=C_{1} \times \ldots \times C_{n}$.

An SLRF mechanism $\mathrm{M}_{\mathrm{SLRF}}$ combined with possible types of the agents $\left(\Theta_{1}, \ldots, \Theta_{n}\right)$, probability density $\phi(\cdot)$, utility functions $\left(u_{1}(\cdot), \ldots, u_{n}(\cdot)\right)$, and description of leader agent $l$ defines a Bayesian Stackelberg game $\Gamma_{s}^{b}$ which gets induced among the agents when the social planner invokes this mechanism as a means to solve the SLRF problem. The induced Bayesian Stackelberg game $\Gamma_{s}^{b}$ is given by

$$
\Gamma_{s}^{b}=\left(\{l\}, N \backslash\{l\},\left(C_{i}\right)_{i \in N},\left(\Theta_{i}\right)_{i \in N}, \phi(\cdot),\left(\bar{u}_{i}\right)_{i \in N}\right)
$$

where $\bar{u}_{i}: C \times \Theta \rightarrow \mathbb{R}$ is the utility function of agent $i$ and is defined in the following manner:

$$
\bar{u}_{i}(c, \theta)=u_{i}\left(g(c), \theta_{i}\right)
$$

where $\Theta=\Theta_{1} \times \cdots \times \Theta_{n}$. In view of the above definition, the trivial scheme of asking the agents to reveal their types, namely, direct revelation mechanism becomes a special case. We define SLRF direct revelation mechanism next.

\section{SLRF Direct Revelation Mechanism}

Definition 3.3: An SLRF direct revelation mechanism $D_{\text {SLRF }}$ corresponding to a SLRF social choice function $f(\cdot)$ is the one in which $C_{i}=\Theta_{i} \forall i \in N$ and $g(\theta)=f(\theta) \forall \theta \in \Theta$.

In the case of direct revelation mechanisms, the action set for each agent is the corresponding type set itself. In the case of an indirect mechanism, each agent is made to announce an action rather than a type. By design, the action announced by the agent is indirectly dependent on the private type of the agent. On the other hand, in a direct mechanism, each agent is asked to reveal his type itself. The first-price and second-price procurement auctions with reserve prices are examples of a direct revelation mechanism for SLRF problem. We now formally describe the various elements of these two direct revelation mechanisms.

\section{E. F-PAR and S-PAR: Examples of SLRF Direct Revelation Mechanisms}

Notice that the agents in both F-PAR and S-PAR have been divided into two levels of hierarchy, $H=\left\{H_{1}, H_{2}\right\}$. The first level of hierarchy consists of a single agent, that is buyer, and the second level of hierarchy consists of the rest of the agents, that is sellers. In both of these examples, the marketplace acts as a social planner. It is easy to see that in both of these examples, the mechanism employed by the social planner is a direct revelation SLRF mechanism. The components of these two SLRF direct revelation mechanisms are listed below.

1) Outcome Set $X$ : An outcome in both these cases may be represented by a vector $x=\left(y_{b}, y_{1}, \ldots\right.$, $\left.y_{n}, t_{b}, t_{1}, \ldots, t_{n}\right)$, where $y_{b}=1$ if the buyer receives the object, $y_{b}=0$ otherwise, and $t_{b}$ is the monetary transfer received by the buyer. Similarly, $y_{i}=-1$ if the seller $i$ is the winner, $y_{i}=0$ otherwise, and $t_{i}$ is the monetary transfer received by the seller $i$. The set of feasible alternatives is then

$$
\begin{aligned}
& X=\left\{\left(y_{b}, y_{1}, \ldots, y_{n}, t_{b}, t_{1}, \ldots, t_{n}\right) \mid\right. \\
& y_{b} \in\{0,1\}, y_{i} \in\{0,-1\}, y_{b}+\sum_{i=1}^{n} y_{i}=0, \\
& \left.t_{i} \in \mathbb{R}, t_{b} \in \mathbb{R}, t_{b}+\sum_{i=1}^{n} t_{i} \leq 0\right\} .
\end{aligned}
$$

2) Utility Function of Buyer $u_{b}(\cdot)$ : The utility function of the buyer is given by

$$
u_{b}\left(x, \theta_{b}\right)=\theta_{b} y_{b}+t_{b} .
$$

3) Utility Function of Sellers $u_{i}(\cdot)$ : The utility function of seller $i$ is given by

$$
u_{i}\left(x, \theta_{i}\right)=\theta_{i} y_{i}+t_{i}
$$

4) Social Choice Function $f(\cdot)$ : The general structure of the social choice function used by the direct mechanism in both F-PAR and S-PAR is

$$
\begin{aligned}
f\left(\theta_{b}, \theta\right)=\left(y_{b}\left(\theta_{b}, \theta\right),\right. & y_{1}\left(\theta_{b}, \theta\right), \ldots, y_{n}\left(\theta_{b}, \theta\right), \\
& \left.t_{b}\left(\theta_{b}, \theta\right), t_{1}\left(\theta_{b}, \theta\right), \ldots, t_{n}\left(\theta_{b}, \theta\right)\right)
\end{aligned}
$$

where $\theta=\left(\theta_{1}, \ldots, \theta_{n}\right)$. Note that $y_{b}(\cdot)$, and $y_{i}(\cdot)$ depend on the winner determination rule, whereas $t_{b}(\cdot)$ and $t_{i}(\cdot)$ depend on the payment rule. Let $\theta_{(k)}$ be the $k$ th smallest element in the set $\left\{\theta_{1}, \ldots, \theta_{n}\right\}$ and $\left(\theta_{-i}\right)_{(k)}$ is the $k$ th smallest element in the set $\left\{\theta_{1}, \ldots, \theta_{i-1}, \theta_{i+1}, \ldots \theta_{n}\right\}$. In view of these definitions, we can define each component of the social choice function. Note that the allocation rule is the same for both F-PAR and S-PAR and, therefore, the definitions of $y_{b}(\cdot)$ and $y_{i}(\cdot)$ are the same for both ${ }^{5}$ :

$$
\begin{aligned}
& y_{b}\left(\theta_{b}, \theta\right)= \begin{cases}0, & \theta_{b}<\theta_{(1)} \\
1, & \text { otherwise }\end{cases} \\
& y_{i}\left(\theta_{b}, \theta\right)= \begin{cases}0, & \theta_{b}<\theta_{(1)} \\
-1, & \theta_{(1)} \leq \theta_{b}, \theta_{i}=\theta_{(1)} . \\
0, & \text { otherwise }\end{cases}
\end{aligned}
$$

It is easy to see that the above definition satisfies the following condition:

$$
y_{b}\left(\theta_{b}, \theta\right)+\sum_{i=1}^{n} y_{i}\left(\theta_{b}, \theta\right)=0 .
$$

The payment rules can be defined as follows. Payment Rule for F-PAR

$$
\begin{aligned}
& t_{b}\left(\theta_{b}, \theta\right)=-\sum_{i=1}^{n} t_{i}\left(\theta_{b}, \theta\right) ; \\
& t_{i}\left(\theta_{b}, \theta\right)=\theta_{i} y_{i}\left(\theta_{b}, \theta\right) .
\end{aligned}
$$

\footnotetext{
${ }^{5}$ Note that two sellers reporting the same type is a zero probability event.
} 
Payment Rule for S-PAR

$$
\begin{aligned}
& t_{b}\left(\theta_{b}, \theta\right)=-\sum_{i=1}^{n} t_{i}\left(\theta_{b}, \theta\right) \\
& t_{i}\left(\theta_{b}, \theta\right)= \begin{cases}\theta_{b} y_{i}\left(\theta_{b}, \theta\right), & \theta_{b}<\left(\theta_{-i}\right)_{(1)} \\
\left(\theta_{-i}\right)_{(1)} y_{i}\left(\theta_{b}, \theta\right), & \text { otherwise }\end{cases}
\end{aligned}
$$

It is easy to see that transfer functions for both F-PAR and S-PAR satisfy the following budget balance property:

$$
t_{b}\left(\theta_{b}, \theta\right)+\sum_{i=1}^{n} t_{i}\left(\theta_{b}, \theta\right)=0 .
$$

In view of the above discussion about direct and indirect SLRF mechanisms, we can say that the social planner can use either an indirect mechanism $M_{\mathrm{SLRF}}$, or a direct mechanism $D_{\mathrm{SLRF}}$ to elicit the information about the leader and the followers' preferences in an indirect or a direct manner, respectively. However, each scheme would induce a game among the leader and the followers, and the social planner is concerned with whether or not the outcome of the game matches with the outcome of the social choice function $f(\theta)$ (if the leader and all the followers had revealed their true types when asked directly). This notion is captured in the following definition.

\section{F. Implementing an SLRF SCF in Bayesian Stackelberg Equilibrium}

Definition 3.4: We say that the mechanism $\mathrm{M}_{\mathrm{SLRF}}=$ $\left(\left(C_{i}\right)_{i \in N}, g(\cdot)\right)$ implements an SLRF social choice function $f(\cdot)$ in Bayesian Stackelberg equilibrium if there is a pure strategy Bayesian Stackelberg equilibrium $s^{*}(\cdot)=\left(s_{l}^{*}(\cdot), t_{-l}^{*}(\cdot)\right)$ of the game $\Gamma_{s}^{b}$ induced by $\mathrm{M}_{\mathrm{SLRF}}$ such that

$$
g\left(s_{l}^{*}\left(\theta_{l}\right),\left(t_{-l}^{*}\left(s_{l}^{*}\left(\theta_{l}\right)\right)\right)\left(\theta_{-l}\right)\right)=f\left(\theta_{l}, \theta_{-l}\right) \quad \forall\left(\theta_{l}, \theta_{-l}\right) \in \Theta .
$$

Using the definition of Bayesian Stackelberg equilibrium, we can say that $s^{*}=\left(s_{l}^{*}, t_{-l}^{*}\right)$ is a pure strategy Bayesian Stackelberg equilibrium of the game $\Gamma_{s}^{b}$ induced by the mechanism $\mathrm{M}_{\mathrm{SLRF}}$ iff it satisfies the following conditions.

Condition 1: Leader Plays a Secure Strategy

$$
\begin{aligned}
& \min _{s_{-l} \in R\left(s_{l}^{*}\left(\theta_{l}\right)\right)} \sum_{\theta_{-l} \in \Theta_{-l}} \phi\left(\theta_{-l} \mid \theta_{l}\right) u_{l}\left(g\left(s_{l}^{*}\left(\theta_{l}\right), s_{-l}\left(\theta_{-l}\right), \theta_{l}\right)\right. \\
& \geq \min _{s_{-l} \in R\left(c_{l}\right)} \sum_{\theta_{-l} \in \Theta_{-l}} \phi\left(\theta_{-l} \mid \theta_{l}\right) u_{l}\left(g\left(c_{l}, s_{-l}\left(\theta_{-l}\right), \theta_{l}\right)\right. \\
& \forall c_{l} \in C_{l}, \quad \forall \theta_{l} \in \Theta_{l}
\end{aligned}
$$

where

$$
\begin{aligned}
& R\left(c_{l}\right)=\left\{s_{-l} \in S_{-l} \mid v_{\theta_{j}}\left(c_{l}, c_{j}, s_{-l, j}\right) \leq v_{\theta_{j}}\left(c_{l}, s_{-l}\right),\right. \\
& \left.\forall c_{j} \in C_{j}, \forall \theta_{j} \in \Theta_{j}, \forall j \in N \backslash\{l\}\right\} \\
& v_{\theta_{j}}\left(c_{l}, s_{-l}\right)=\sum_{\theta_{-j} \in \Theta_{-j}} \phi\left(\theta_{-j} \mid \theta_{j}\right) u_{j} \\
& \quad \times\left(g\left(c_{l}, s_{-l}\left(\theta_{-l}\right), \theta_{j}\right)\right. \\
& v_{\theta_{j}}\left(c_{l}, c_{j}, s_{-l, j}\right)=\sum_{\theta_{-j} \in \Theta_{-j}} \phi\left(\theta_{-j} \mid \theta_{j}\right) u_{j} \\
& \quad \times\left(g\left(c_{l}, c_{j}, s_{-l, j}\left(\theta_{-l, j}\right), \theta_{j}\right) .\right.
\end{aligned}
$$

Condition 2: Followers Play an Optimal Response

$$
t_{-l}^{*}\left(s_{l}^{*}\left(\theta_{l}\right)\right) \in R\left(s_{l}^{*}\left(\theta_{l}\right)\right) \quad \forall \theta_{l} \in \Theta_{l} .
$$

What follows is an important proposition which establishes the relationship between dominant strategy equilibrium and Bayesian Stackelberg equilibrium. The corollary to this proposition is extremely important in the sense that it connects the classical mechanism design theory in the absence of hierarchical decision making with the theory of mechanism design for hierarchical decision making.

Proposition 3.1: Let $s^{d}(\cdot)=\left(s_{1}^{d}(\cdot), \ldots, s_{n}^{d}(\cdot)\right)$ be a weakly dominant strategy equilibrium of the Bayesian game $\Gamma^{b}$ induced by the mechanism $\mathrm{M}=\left(\left(C_{i}\right)_{i \in N}, g(\cdot)\right)$. Consider the same set of players and a Bayesian Stackelberg game $\Gamma_{s}^{b}$, which gets induced among them when we designate one of the agents as leader and the rest of the agents as followers. That is, we convert the mechanism $\mathrm{M}$ into $\mathrm{M}_{\mathrm{SLRF}}=\left(C_{l},\left(C_{i}\right)_{i \in N \backslash\{l\}}, g(\cdot)\right)$. Then, $s^{*}(\cdot)=\left(s_{l}^{*}(\cdot), t_{-l}^{*}(\cdot)\right)$ is a pure strategy Bayesian Stackelberg equilibrium of the game $\Gamma_{s}^{b}$, where $s^{d}(\cdot)=$ $\left(s_{1}^{d}(\cdot), \ldots, s_{n}^{d}(\cdot)\right)=\left(s_{l}^{d}(\cdot), s_{-l}^{d}(\cdot)\right)$ and

$$
\begin{aligned}
s_{l}^{*}\left(\theta_{l}\right) & =s_{l}^{d}\left(\theta_{l}\right), \quad \forall \theta_{l} \in \Theta_{l} \\
t_{-l}^{*}\left(s_{l}^{*}\left(\theta_{l}\right)\right)\left(\theta_{-l}\right)= & s_{-l}^{d}\left(\theta_{-l}\right), \\
& \forall \theta_{l} \in \Theta_{l}, \forall \theta_{-l} \in \Theta_{-l} .
\end{aligned}
$$

Corollary 3.1: Let the mechanism $\mathrm{M}=\left(\left(C_{i}\right)_{i \in N}, g(\cdot)\right)$ implement the social choice function $f(\cdot)$ in dominant strategy equilibrium. Then, the mechanism $\mathrm{M}_{\mathrm{SLRF}}=$ $\left(C_{l},\left(C_{i}\right)_{i \in N \backslash\{l\}}, g(\cdot)\right)$ will also implement the $f(\cdot)$ in Bayesian Stackelberg equilibrium.

Proof: Consider the following definition of dominant strategy equilibrium:

$$
\begin{aligned}
& u_{i}\left(g\left(s_{i}^{d}\left(\theta_{i}\right), s_{-i}\left(\theta_{-i}\right)\right), \theta_{i}\right) \\
& \quad \geq u_{i}\left(g\left(s_{i}^{\prime}\left(\theta_{i}\right), s_{-i}\left(\theta_{-i}\right)\right), \theta_{i}\right) \\
& \quad \forall i \in N, \forall \theta_{i} \in \Theta_{i}, \\
& \quad \forall \theta_{-i} \in \Theta_{-i}, \forall s_{i}^{\prime}(\cdot) \in S_{i}, \forall s_{-i}(\cdot) \in S_{-i} .
\end{aligned}
$$

We need to show that condition (26) together with conditions (24) and (25) imply the conditions (19) and (23). This will essentially prove the proposition.

Part I: First, we prove the condition (23). For this, we start with the condition (26). Note that for any follower $j \in N$, the condition (26) implies that

$$
\begin{gathered}
u_{j}\left(g\left(s_{j}^{d}\left(\theta_{j}\right), s_{-j}\left(\theta_{-j}\right)\right), \theta_{j}\right) \\
\geq u_{j}\left(g\left(s_{j}^{\prime}\left(\theta_{j}\right), s_{-j}\left(\theta_{-j}\right), \theta_{j}\right)\right. \\
\forall \theta_{j} \in \Theta_{j}, \forall \quad \theta_{-j} \in \Theta_{-j}, \\
\forall s_{j}^{\prime} \in S_{j}, \forall \quad s_{-j} \in S_{-j} \\
\Rightarrow \quad u_{j}\left(g\left(s_{j}^{d}\left(\theta_{j}\right), s_{-j}\left(\theta_{-j}\right)\right), \theta_{j}\right) \\
\geq u_{j}\left(g\left(c_{j}, s_{-j}\left(\theta_{-j}\right), \theta_{j}\right)\right. \\
\forall \theta_{j} \in \Theta_{j}, \forall \quad \theta_{-j} \in \Theta_{-j}, \\
\forall c_{j} \in C_{j}, \forall \quad s_{-j} \in S_{-j}
\end{gathered}
$$




$$
\begin{aligned}
& \Rightarrow \quad \sum_{\theta_{-j} \in \Theta_{-j}} \phi\left(\theta_{-j} \mid \theta_{j}\right) u_{j} \\
& \times\left(g\left(s_{j}^{d}\left(\theta_{j}\right), s_{-j}\left(\theta_{-j}\right)\right), \theta_{j}\right) \\
& \geq \sum_{\theta_{-j} \in \Theta_{-j}} \phi\left(\theta_{-j} \mid \theta_{j}\right) u_{j}\left(g\left(c_{j}, s_{-j}\left(\theta_{-j}\right), \theta_{j}\right)\right. \\
& \forall \theta_{j} \in \Theta_{j}, \forall \quad c_{j} \in C_{j}, \forall \quad s_{-j} \in S_{-j} \\
& \Rightarrow \quad \sum_{\theta_{-j} \in \Theta_{-j}} \phi\left(\theta_{-j} \mid \theta_{j}\right) u_{j} \\
& \times\left(g\left(s_{j}^{d}\left(\theta_{j}\right), s_{l}^{*}\left(\theta_{l}\right), s_{-l, j}\left(\theta_{-l, j}\right)\right), \theta_{j}\right) \\
& \geq \sum_{\theta_{-j} \in \Theta_{-j}} \phi\left(\theta_{-j} \mid \theta_{j}\right) u_{j} \\
& \times\left(g\left(c_{j}, s_{l}^{*}\left(\theta_{l}\right), s_{-l, j}\left(\theta_{-l, j}\right), \theta_{j}\right),\right. \\
& \forall \theta_{j} \in \Theta_{j}, \forall \quad c_{j} \in C_{j}, \\
& \forall \theta_{l} \in \Theta_{l}, \forall \quad s_{-l, j} \in S_{-l, j} \\
& \sum_{\theta_{-j} \in \Theta_{-j}} \phi\left(\theta_{-j} \mid \theta_{j}\right) u_{j} \\
& \quad \times\left(g\left(s_{j}^{d}\left(\theta_{j}\right), s_{l}^{*}\left(\theta_{l}\right), s_{-l, j}^{d}\left(\theta_{-l, j}\right)\right), \theta_{j}\right) \\
& \geq \sum_{\theta_{-j} \in \Theta_{-j}} \phi \\
& \quad \times\left(\theta_{-j} \mid \theta_{j}\right) u_{j}\left(g\left(c_{j}, s_{l}^{*}\left(\theta_{l}\right), s_{-l, j}^{d}\left(\theta_{-l, j}\right), \theta_{j}\right),\right. \\
& \forall \theta_{j} \in \Theta_{j}, \forall \quad c_{j} \in C_{j}, \\
& \forall \theta_{l} \in \Theta_{l}, \forall \quad s_{-l, j} \in S_{-l, j} .
\end{aligned}
$$

Using relation (25), the above can be written as

$$
\begin{aligned}
& \sum_{\theta_{-j} \in \Theta_{-j}} \phi\left(\theta_{-j} \mid \theta_{j}\right) u_{j} \\
& \times\left(g\left(s_{l}^{*}\left(\theta_{l}\right), t_{-l}^{*}\left(s_{l}^{*}\left(\theta_{l}\right)\right)\left(\theta_{-l}\right)\right), \theta_{j}\right) \\
& \geq \sum_{\theta_{-j} \in \Theta_{-j}} \phi\left(\theta_{-j} \mid \theta_{j}\right) u_{j}\left(g \left(s_{l}^{*}\left(\theta_{l}\right), c_{j}, t_{-l, j}^{*}\right.\right. \\
&\left.\left.\times\left(s_{l}^{*}\left(\theta_{l}\right)\right)\left(\theta_{-l, j}\right)\right), \theta_{j}\right), \\
& \forall \forall \theta_{j} \in \Theta_{j} \forall \quad c_{j} \in C_{j}, \forall \quad \theta_{l} \in \Theta_{l} \\
& \Rightarrow \quad t_{-l}^{*}\left(s_{l}^{*}\left(\theta_{l}\right)\right) \in R\left(s_{l}^{*}\left(\theta_{l}\right)\right), \quad \forall \theta_{l} \in \Theta_{l} .
\end{aligned}
$$

This is precisely the condition (23).

Part II: Now, we take up proving the condition (19). For this, we start with condition (26). Note that for the leader $l \in N$, the condition (26) implies that

$$
\begin{gathered}
u_{l}\left(g\left(s_{l}^{d}\left(\theta_{l}\right), s_{-l}\left(\theta_{-l}\right)\right), \theta_{l}\right) \\
\geq u_{l}\left(g\left(s_{l}^{\prime}\left(\theta_{l}\right), s_{-l}\left(\theta_{-l}\right), \theta_{l}\right)\right. \\
\forall \theta_{l} \in \Theta_{l}, \forall \theta_{-l} \in \Theta_{-l}, \forall s_{l}^{\prime} \in S_{l}, \forall s_{-l} \in S_{-l} \\
\Rightarrow \quad \sum_{\theta_{-l} \in \Theta_{-l}} \phi\left(\theta_{-l} \mid \theta_{l}\right) u_{l}\left(g\left(s_{l}^{d}\left(\theta_{l}\right), s_{-l}\left(\theta_{-l}\right)\right), \theta_{l}\right) \\
\geq \sum_{\theta_{-l} \in \Theta_{-l}} \phi\left(\theta_{-l} \mid \theta_{l}\right) u_{l}\left(g\left(s_{l}^{\prime}\left(\theta_{l}\right), s_{-l}\left(\theta_{-l}\right), \theta_{l}\right)\right. \\
\forall \theta_{l} \in \Theta_{l}, \forall s_{l}^{\prime} \in S_{l}, \forall s_{-l} \in S_{-l} .
\end{gathered}
$$

Substituting the values from (24), we get the following relation:

$$
\begin{gathered}
\sum_{\theta_{-l} \in \Theta_{-l}} \phi\left(\theta_{-l} \mid \theta_{l}\right) u_{l}\left(g\left(s_{l}^{*}\left(\theta_{l}\right), s_{-l}\left(\theta_{-l}\right)\right), \theta_{l}\right) \\
\geq \sum_{\theta_{-l} \in \Theta_{-l}} \phi\left(\theta_{-l} \mid \theta_{l}\right) u_{l}\left(g\left(s_{l}^{\prime}\left(\theta_{l}\right), s_{-l}\left(\theta_{-l}\right), \theta_{l}\right)\right. \\
\forall \theta_{l} \in \Theta_{l}, \forall s_{l}^{\prime} \in S_{l}, \forall s_{-l} \in S_{-l} \\
\sum_{\theta_{-l} \in \Theta_{-l}} \phi\left(\theta_{-l} \mid \theta_{l}\right) u_{l}\left(g\left(s_{l}^{*}\left(\theta_{l}\right), s_{-l}\left(\theta_{-l}\right)\right), \theta_{l}\right) \\
\geq \sum_{\theta_{-l} \in \Theta_{-l}} \phi\left(\theta_{-l} \mid \theta_{l}\right) u_{l}\left(g\left(c_{l}, s_{-l}\left(\theta_{-l}\right), \theta_{l}\right)\right. \\
\forall \theta_{l} \in \Theta_{l}, \forall c_{l} \in C_{l}, \forall s_{-l} \in S_{-l} .
\end{gathered}
$$

It is easy to see that $R\left(c_{l}\right)$ is the same for all $c_{l} \in C_{l}$ and it contains only those pure strategy profiles of the followers which are part of a weakly dominant strategy equilibrium in the game $\Gamma^{b}$. Therefore, the above inequality can be written as

$$
\begin{gathered}
\min _{s_{-l} \in R\left(s_{l}^{*}\left(\theta_{l}\right)\right)} \sum_{\theta_{l} \in \Theta_{l}} \phi\left(\theta_{-l} \mid \theta_{l}\right) u_{l}\left(g\left(s_{l}^{*}\left(\theta_{l}\right), s_{-l}\left(\theta_{-l}\right)\right), \theta_{l}\right) \\
\geq \min _{s_{-l} \in R\left(c_{l}\right)} \sum_{\theta_{l} \in \Theta_{l}} \phi\left(\theta_{-l} \mid \theta_{l}\right) u_{l}\left(g\left(c_{l}, s_{-l}\left(\theta_{-l}\right), \theta_{l}\right)\right.
\end{gathered}
$$$$
\forall \theta_{l} \in \Theta_{l}, \forall c_{l} \in C_{l}
$$

which is precisely the condition (19). (Q.E.D.).

\section{G. Bayesian Stackelberg Incentive Compatibility (Basic)}

Unlike the dominant strategy and Bayesian incentive compatibility, the incentive compatibility of a social choice function for an SLRF problem needs to be defined separately for the leader and the followers.

1) BaSIC for the Leader:

Definition 3.5: An SCF $f(\cdot)$ is said to be Bayesian Stackelberg incentive compatible (BaSIC) for the leader (or truthfully implementable in Bayesian Stackelberg equilibrium for the leader) if the direct revelation mechanism $\mathrm{D}_{\mathrm{SLRF}}=\left(\left(\Theta_{i}\right)_{i \in N}, f(\cdot)\right)$ has a Bayesian Stackelberg equilibrium $s^{*}=\left(s_{1}^{*}, t_{-l}^{*}\right)$ in which

$$
s_{l}^{*}\left(\theta_{l}\right)=\theta_{l}, \forall \theta_{l} \in \Theta_{l} .
$$

That is, truth revelation is a Bayesian Stackelberg equilibrium strategy for the leader in the game induced by $\mathrm{D}_{\mathrm{SLRF}}$. Following is a necessary and sufficient condition for an $\operatorname{SCF} f(\cdot)$ to be BaSIC for the leader

$$
\begin{aligned}
& \min _{s_{-l} \in R\left(\theta_{l}\right)} \sum_{\theta_{-l} \in \Theta_{-l}} \phi_{l}\left(\theta_{-l} \mid \theta_{l}\right) u_{l}\left(f\left(\theta_{l}, s_{-l}\left(\theta_{-l}\right)\right), \theta_{l}\right) \\
& \geq \min _{s_{-l}^{\prime} \in R\left(\theta_{l}^{\prime}\right)} \sum_{\theta_{-l} \in \Theta_{-l}} \phi_{l}\left(\theta_{-l} \mid \theta_{l}\right) u_{l}\left(f\left(\theta_{l}^{\prime}, s_{-l}^{\prime}\left(\theta_{-l}\right)\right), \theta_{l}\right) \\
& \forall \theta_{l} \in \Theta_{l}, \forall \theta_{l}^{\prime} \in \Theta_{l}
\end{aligned}
$$


where

$$
\begin{aligned}
& R\left(\theta_{l}\right)=\left\{s_{-l} \in S_{-l} \mid v_{\theta_{j}}\left(\theta_{l}, \hat{\theta_{j}}, s_{-l, j}\right) \leq v_{\theta_{j}}\left(\theta_{l}, s_{-l}\right),\right. \\
& \left.\quad \forall \hat{\theta_{j}} \in \Theta_{j}, \forall \theta_{j} \in \Theta_{j}, \forall j \in N \backslash\{l\}\right\} \\
& v_{\theta_{j}}\left(\theta_{l}, s_{-l}\right)=\sum_{\theta_{-j} \in \Theta_{-j}} \phi_{j} \\
& \quad \times\left(\theta_{-j} \mid \theta_{j}\right) u_{j}\left(f\left(\theta_{l}, s_{-l}\left(\theta_{-l}\right), \theta_{j}\right)\right. \\
& v_{\theta_{j}}\left(\theta_{l}, \hat{\theta_{j}}, s_{-l, j}\right)=\sum_{\theta_{-j} \in \Theta_{-j}} \phi_{j}\left(\theta_{-j} \mid \theta_{j}\right) u_{j} \\
& \quad \times\left(f\left(\theta_{l}, \hat{\theta_{j}}, s_{-l, j}\left(\theta_{-l, j}\right), \theta_{j}\right) .\right.
\end{aligned}
$$

Recall that the implicit assumption here is that all the type sets $\Theta_{i}, i \in N$ are finite. If the sets $\Theta_{i}, i \in N$ are infinite, then the above condition will transform to the following condition:

$$
\begin{aligned}
& \quad \inf _{s_{-l} \in R\left(\theta_{l}\right)} E_{\theta_{-l}}\left[u_{l}\left(f\left(\theta_{l}, s_{-l}\left(\theta_{-l}\right)\right), \theta_{l}\right) \mid \theta_{l}\right] \\
& \quad \geq \inf _{s_{-l}^{\prime} \in R\left(\theta_{l}^{\prime}\right)} E_{\theta_{-l}}\left[u_{l}\left(f\left(\theta_{l}^{\prime}, s_{-l}^{\prime}\left(\theta_{-l}\right)\right), \theta_{l}\right) \mid \theta_{l}\right], \forall \theta_{l}, \theta_{l}^{\prime} \in \Theta_{l}
\end{aligned}
$$

assuming that the infimum is attained for some $s_{-l}$.

2) Basic for the Followers:

Definition 3.6: An SCF $f(\cdot)$ is said to be Bayesian Stackelberg incentive compatible (BaSIC) for the followers (or truthfully implementable in Bayesian Stackelberg equilibrium for the followers) if the direct revelation mechanism $\mathrm{D}_{\mathrm{SLRF}}=$ $\left(\left(\Theta_{i}\right)_{i \in N}, f(\cdot)\right)$ has a Bayesian Stackelberg equilibrium $s^{*}=$ $\left(s_{l}^{*}, t_{-l}^{*}\right)$ in which

$$
t_{-l}^{*}\left(\theta_{l}\right)=\left(\left(s_{j}^{*}\right)_{j \in N \backslash\{l\}}\right), \forall \theta_{l} \in \Theta_{l}
$$

where

$$
s_{j}^{*}\left(\theta_{j}\right)=\theta_{j}, \forall \theta_{j} \in \Theta_{j}, \forall j \in N \backslash\{l\} .
$$

That is, truth revelation is a Bayesian Stackelberg equilibrium strategy for the followers in the game induced by $\mathrm{D}_{\mathrm{SLRF}}$. Following is a necessary and sufficient condition for an SCF $f(\cdot)$ to be BaSIC for the followers:

$$
\begin{aligned}
& \sum_{\theta_{-j} \in \Theta_{-j}} \phi_{j}\left(\theta_{-j} \mid \theta_{j}\right) u_{j}\left(f\left(\theta_{l}, \theta_{j}, \theta_{-l, j}\right), \theta_{j}\right) \\
& \geq \sum_{\theta_{-j} \in \Theta_{-j}} \phi_{j}\left(\theta_{-j} \mid \theta_{j}\right) u_{j}\left(f\left(\theta_{l}, \theta_{j}^{\prime}, \theta_{-l, j}\right), \theta_{j}\right) \\
& \forall \theta_{j}, \theta_{j}^{\prime} \in \Theta_{j}, \forall \theta_{l} \in \Theta_{l}, \forall j \in(N \backslash\{l\}) .
\end{aligned}
$$

Recall that the implicit assumption here is that all the type sets $\Theta_{i}, i \in N$ are finite sets. If the sets $\Theta_{i}, i \in N$ are infinite, then the above condition will reduce to the following condition:

$$
\begin{aligned}
& E_{\theta_{-j}}\left[u_{j}\left(f\left(\theta_{l}, \theta_{j}, \theta_{-l, j}\right), \theta_{j}\right) \mid \theta_{j}\right] \\
& \geq E_{\theta_{-j}}\left[u_{j}\left(f\left(\theta_{l}, \theta_{j}^{\prime}, \theta_{-l, j}\right), \theta_{j}\right) \mid \theta_{j}\right] \\
& \forall \theta_{j}^{\prime} \in \Theta_{j}, \forall \theta_{l} \in \Theta_{l}, \forall j \in(N \backslash\{l\}) .
\end{aligned}
$$

3) Bayesian Stackelberg Incentive Compatibility of a Social Choice Function:

Definition 3.7: We call an SCF $f(\cdot)$ to be Bayesian Stackelberg incentive compatible (BaSIC) if it is BaSIC for both the leader and the followers.

The following proposition provides a sufficient condition for BaSIC of a social choice function in the presence of hierarchical decision making.

Proposition 3.2: If an SCF $f(\cdot)$ is dominant strategy incentive compatible then it will be BaSIC.

Proof: Let $f(\cdot)$ be a dominant strategy incentive compatible SCF. By the definition of dominant strategy incentive compatibility, we can say that the direct revelation mechanism $\mathrm{D}=$ $\left(\left(\Theta_{i}\right)_{i \in N}, f(\cdot)\right)$ has a dominant strategy equilibrium $s^{d}(\cdot)=$ $\left(s_{1}^{d}(\cdot), \ldots, s_{n}^{d}(\cdot)\right)$ in which

$$
s_{i}^{d}\left(\theta_{i}\right)=\theta_{i}, \forall \theta_{i} \in \Theta_{i}, \forall i \in N
$$

Invoking Proposition 3.1, we can say that the direct revelation mechanism $\mathrm{D}_{\mathrm{SLRF}}=\left(\left(\Theta_{i}\right)_{i \in N}, f(\cdot)\right)$ has a Bayesian Stackelberg equilibrium $s^{*}=\left(s_{l}^{*}, t_{-l}^{*}\right)$ in which

$$
\begin{aligned}
s_{l}^{*}\left(\theta_{l}\right) & =s_{l}^{d}\left(\theta_{l}\right), \forall \theta_{l} \in \Theta_{l} \\
t_{-l}^{*}\left(s_{l}^{*}\left(\theta_{l}\right)\right)\left(\theta_{-l}\right) & =s_{-l}^{d}\left(\theta_{-l}\right), \forall \theta_{l} \in \Theta_{l}, \forall \theta_{-l} \in \Theta_{-l} .
\end{aligned}
$$

Substituting the values of (37) in (38) and (38), we get the following Bayesian Stackelberg equilibrium of the direct revelation mechanism $\mathrm{D}_{\mathrm{SLRF}}$, which suffices to prove that $f(\cdot)$ is BaSIC

$$
\begin{aligned}
s_{l}^{*}\left(\theta_{l}\right) & =\theta_{l}, \forall \theta_{l} \in \Theta_{l} \\
t_{-l}^{*}\left(s_{l}^{*}\left(\theta_{l}\right)\right)\left(\theta_{-l}\right) & =\theta_{-l}, \forall \theta_{l} \in \Theta_{l}, \forall \theta_{-l} \in \Theta_{-l}
\end{aligned}
$$

(Q.E.D.).

\section{H. Revelation Principle for Followers}

Analogous to the revelation principles for dominant strategy equilibrium and Bayesian Nash equilibrium, we also have the revelation principle for Bayesian Stackelberg equilibrium.

Proposition 3.3: (Revelation Principle for Followers): Suppose that there exists a mechanism $\mathrm{M}_{\mathrm{SLRF}}=$ $\left(C_{1}, \ldots, C_{n}, g(\cdot)\right)$ that implements the SCF $f(\cdot)$ in Bayesian Stackelberg equilibrium. Then, $f(\cdot)$ is BaSIC for the followers.

Proof: If $\mathrm{M}_{\mathrm{SLRF}}=\left(C_{1}, \ldots, C_{n}, g(\cdot)\right)$ implements SCF $f(\cdot)$ in Bayesian Stackelberg equilibrium, then there exists a pure strategy Bayesian Stackelberg equilibrium $s^{*}=\left(s_{l}^{*}, t_{-l}^{*}\right)$ of the game $\Gamma_{s}^{b}$ induced by M $M_{\text {SLRF }}$ such that $g\left(s_{l}^{*}\left(\theta_{l}\right),\left(t_{-l}^{*}\left(s_{l}^{*}\left(\theta_{l}\right)\right)\right)\left(\theta_{-l}\right)\right)=f\left(\theta_{l}, \theta_{-l}\right), \forall\left(\theta_{l}, \theta_{-l}\right) \in \Theta$. Such an equilibrium must satisfy conditions (19)-(23). Also, we have seen that a SCF $f(\cdot)$ is BaSIC for the followers iff it satisfies the condition (35). Therefore, in order to prove the above revelation principle, we must show that the conditions 
(19)-(23) implies the conditions (35). For this, we start with condition (23) and rewrite it in following form:

$$
\begin{aligned}
& t_{-l}^{*}\left(s_{l}^{*}\left(\theta_{l}\right)\right) \in R\left(s_{l}^{*}\left(\theta_{l}\right)\right), \forall \theta_{l} \in \Theta_{l} \\
& \Rightarrow \quad \sum_{\theta_{-j} \in \Theta_{-j}} \phi_{j} \times\left(\theta_{-j} \mid \theta_{j}\right) u_{j} \\
& \times\left(g\left(s_{l}^{*}\left(\theta_{l}\right), c_{j}, t_{-l, j}^{*}\left(s_{l}^{*}\left(\theta_{l}\right)\right)\left(\theta_{-l, j}\right), \theta_{j}\right)\right. \\
& \leq \sum_{\theta_{-j} \in \Theta_{-j}} \phi_{j}\left(\theta_{-j} \mid \theta_{j}\right) u_{j} \\
& \quad \times\left(g_{l}^{*}\left(\theta_{l}\right), t_{-l}^{*}\left(s_{l}^{*}\left(\theta_{l}\right)\right)\left(\theta_{-l}\right), \theta_{j}\right), \\
& \forall c_{j} \in C_{j}, \forall \theta_{j} \in \Theta_{j}, \forall \theta_{l} \in \Theta_{l}, \forall j \in N \backslash\{l\} \\
& \quad \sum_{\theta-j \in \Theta_{-j}} \phi_{j}\left(\theta_{-j} \mid \theta_{j}\right) u_{j} \\
& \quad \times\left(g\left(s_{l}^{*}\left(\theta_{l}\right), t_{-l}^{*}\left(s_{l}^{*}\left(\theta_{l}\right)\right)\left(\theta_{-l, j}, \hat{\theta_{j}}\right), \theta_{j}\right)\right. \\
& \leq \sum_{\theta_{-j} \in \Theta_{-j}} \phi_{j}\left(\theta_{-j} \mid \theta_{j}\right) u_{j} \\
& \quad \times\left(g\left(s_{l}^{*}\left(\theta_{l}\right), t_{-l}^{*}\left(s_{l}^{*}\left(\theta_{l}\right)\right)\left(\theta_{-l}\right), \theta_{j}\right),\right. \\
& \forall \hat{\theta}_{j} \in \Theta_{j}, \forall \theta_{j} \in \Theta_{j}, \forall \theta_{l} \in \Theta_{l}, \forall j \in N \backslash\{l\} .
\end{aligned}
$$

Using the fact that $g\left(s_{l}^{*}\left(\theta_{l}\right),\left(t_{-l}^{*}\left(s_{l}^{*}\left(\theta_{l}\right)\right)\right)\left(\theta_{-l}\right)\right)=$ $f\left(\theta_{l}, \theta_{-l}\right) \forall\left(\theta_{l}, \theta_{-l}\right) \in \Theta$, we can write the above inequality in the following form:

$$
\begin{aligned}
& \sum_{\theta_{-j} \in \Theta_{-j}} \phi_{j}\left(\theta_{-j} \mid \theta_{j}\right) u_{j}\left(f\left(\theta_{l}, \theta_{j}^{\prime}, \theta_{-l, j}\right), \theta_{j}\right) \\
& \quad \leq \sum_{\theta_{-j} \in \Theta_{-j}} \phi_{j}\left(\theta_{-j} \mid \theta_{j}\right) u_{j}\left(f\left(\theta_{l}, \theta_{j}, \theta_{-l, j}\right), \theta_{j}\right) \\
& \forall \theta_{j} \in \Theta_{j} \forall \theta_{j}^{\prime} \in \Theta_{j} \forall \theta_{l} \in \Theta_{l} \forall j \in(N \backslash\{l\}) .
\end{aligned}
$$

This is essentially the condition (35). (Q.E.D.).

\section{Application to Design of Procurement Auctions WITH RESERVE PRICES}

We now show that the theory of mechanism design for SLRF problems, developed in the previous section, can be effectively used for designing incentive compatible SLRF mechanisms. For this, we investigate the Bayesian Stackelberg incentive compatibility of the social choice functions used in the first-price and second-price procurement auctions with reserve prices (F-PAR and S-PAR, respectively) by modeling both these auctions as SLRF mechanisms. The results obtained in this section are available in the literature for the case of reserve price auctions for selling. See Krishna [11], Myerson [12], and Maskin and Riley [13]. Our results are for procurement auctions and are independently derived using an elegant and natural SLRF approach to the problem. Also, the derivation of results in this section crucially uses Proposition 3.2 which, in turn, uses Proposition 3.1.

We first make certain standard assumptions regarding F-PAR and S-PAR mechanisms. A comprehensive discussion about these assumptions can be found in [14]-[16].

(A1) Risk Neutral Bidders: The buyer and all the $n$ sellers are risk neutral. This essentially implies that the utility functions are linear.

(A2) Independent Private Value (IPV) Model: Each individual, that is buyer as well as sellers, precisely knows the value of the object to himself, but does not know the value of the object to other individuals. Each individual perceives any other individual's valuation as a draw from some probability distribution. Similarly, he or she knows that the other individuals regard his or her own valuation as a draw from some probability distribution. More precisely, for seller $i, i=1,2, \ldots, n$, there is some probability distribution $\Phi_{i}(\cdot)$ from which he draws his valuation $\theta_{i}$. Similarly, the buyer draws her own valuation $\theta_{b}$ from some probability distribution $\Phi_{b}(\cdot)$. Any individual's valuation is statistically independent from any other individual's valuation. That is, $\Phi_{i}(\cdot), i=1,2, \ldots, n$ and $\Phi_{b}(\cdot)$ are mutually independent. The private values of the object to the sellers and the buyer, that is $\theta_{i}$ and $\theta_{b}$, can be viewed as their types. Let $\Theta_{i}, i=1,2, \ldots, n$ and $\Theta_{b}$ denote the set of all possible types of the sellers and buyer, respectively. This implies that $\Phi_{i}(\cdot), i=1,2, \ldots, n$ and $\Phi_{b}(\cdot)$ are probability distribution functions on $\Theta_{i}, i=1,2, \ldots, n$ and $\Theta_{b}$, respectively.

(A3) Symmetry Among Sellers: The sellers are symmetric in the following sense:

- $\Theta_{1}=\Theta_{2}=\ldots=\Theta_{n}=\Theta_{\mathrm{PAR}}$.

- $\Phi_{1}(\cdot)=\Phi_{2}(\cdot)=\ldots=\Phi_{n}(\cdot)=\Phi(\cdot)$.

We also assume that $\Theta_{b}=\Theta_{\mathrm{PAR}}$ and $\Phi_{b}(\cdot)=\Phi(\cdot)$.

(A4) Properties of $\Phi(\cdot)$ and $\Theta_{\mathrm{PAR}}$ : We assume that $F(\cdot)$ and $\Theta_{\mathrm{PAR}}$ satisfy the following properties.

- $\Theta_{\mathrm{PAR}}=[\underline{\theta}, \bar{\theta}]$.

- $\underline{\theta}>0$.

- $\Phi(\cdot)$ is twice continuously differentiable.

- $\phi(\theta)=\Phi^{\prime}(\theta)>0 ; \forall \underline{\theta} \leq \theta \leq \bar{\theta}$.

Using the above assumptions, we derive the following Theorem 4.1 and Theorem 4.2, the main results of this paper, which summarize the incentive compatibility property of the social choice functions used in F-PAR and S-PAR, respectively. As already stated, these results are available in the literature for the case of a forward auction for selling a single indivisible item-see Krishna [11], Myerson [12], and Maskin and Riley [13]. The results we derive are for the case of procurement and use a natural approach based on SLRF mechanisms.

Theorem 4.1: Under the assumptions A1-A4:

1) The SCF used in first-price procurement auction with reserve prices, which is given by (13), (14), and (16) is neither BaSIC for the buyer nor BaSIC for the sellers. The Bayesian Stackelberg equilibrium of the Bayesian Stackelberg game induced by this function among the sellers and the buyer is given by $s^{*}=\left(s_{b}^{*}, t_{-b}^{*}\right)$ where

1.1) $s_{b}^{*}(\cdot)$ is the solution of the following equation

$$
\theta_{b}=s_{b}^{*}\left(\theta_{b}\right)+\frac{\Phi\left(s_{b}^{*}\left(\theta_{b}\right)\right)}{\phi\left(s_{b}^{*}\left(\theta_{b}\right)\right)} .
$$

1.2) $t_{-b}^{*}\left(\hat{\theta_{b}}\right)=\left(s^{*}(\cdot), \ldots, s^{*}(\cdot)\right) \forall \hat{\theta_{b}} \in \Theta_{b}=[\underline{\theta}, \bar{\theta}]$, where

$$
s^{*}\left(\theta_{i}\right)=\left\{\begin{array}{ll}
\theta_{i}, \quad \theta_{i} \in\left[\hat{\theta_{b}}, \bar{\theta}\right] \\
\int_{i}+\frac{\hat{\theta_{i}}[1-\Phi(x)]^{n-1} d x}{\left[1-\Phi\left(\theta_{i}\right)\right]^{n-1}}, & \theta_{i} \in\left[\underline{\theta}, \hat{\theta_{b}}\right]
\end{array} .\right.
$$

2) For an announced reserve price of $\hat{\theta_{b}}$ by the buyer, the expected payoff (utility) $U_{i}\left(\hat{\theta}_{b} \mid \theta_{i}\right)$ and the expected payment 
$R_{i}\left(\hat{\theta}_{b} \mid \theta_{i}\right)$ received by any seller $i$, when his actual type is $\theta_{i}$, turns out to be the following:

$$
\begin{array}{r}
U_{i}\left(\hat{\theta_{b}} \mid \theta_{i}\right)= \begin{cases}0, & \theta_{i} \in\left[\hat{\theta_{b}}, \bar{\theta}\right] \\
\int_{\theta_{i}}[1-\Phi(x)]^{n-1} d x, & \theta_{i} \in\left[\underline{\theta}, \hat{\theta_{b}}\right]\end{cases} \\
R_{i}\left(\hat{\theta_{b}} \mid \theta_{i}\right)= \begin{cases}0, & \theta_{i} \in\left[\hat{\theta_{b}}, \bar{\theta}\right] \\
\left\{\theta_{i}\left[1-\Phi\left(\theta_{i}\right)\right]^{n-1}\right. & \theta_{i} \in\left[\underline{\theta}, \hat{\theta_{b}}\right] \\
\left.+U_{i}\left(\hat{\theta_{b}} \mid \theta_{i}\right)\right\}, & \end{cases}
\end{array}
$$

3) When the actual type of the buyer is $\theta_{b}$ and she announces a reserve price of $\hat{\theta}_{b}$, his expected payoff (utility) $U_{b}\left(\hat{\theta}_{b} \mid \theta_{b}\right)$ and the expected payment $R_{b}\left(\hat{\theta_{b}} \mid \theta_{b}\right)$ made by him turn out to be the following:

$$
\begin{aligned}
& U_{b}\left(\hat{\theta_{b}} \mid \theta_{b}\right)=\left(\theta_{b}-\hat{\theta_{b}}\right) \Phi_{\Theta_{(1)}}\left(\hat{\theta_{b}}\right)+\int_{x=\underline{\theta}}^{\hat{\theta_{b}}}\left[\Phi_{\Theta_{(2)}}(x)\right] d x \\
& R_{b}\left(\hat{\theta_{b}} \mid \theta_{b}\right)=\theta_{b} \Phi_{\Theta_{(1)}}\left(\hat{\theta_{b}}\right)-U_{b}\left(\hat{\theta_{b}} \mid \theta_{b}\right) .
\end{aligned}
$$

\section{Corollary 4.1:}

1) The optimal reserve price strategy of the buyer in F-PAR when all the sellers draw their types independently from the uniform distribution over the set $[0,1]$ is given by $s_{b}^{*}\left(\theta_{b}\right)=\theta_{b} / 2$.

2) If $\hat{\theta}_{b}=\bar{\theta}$, then F-PAR will reduce to the traditional firstprice procurement auction with no reserve price. Further, if the sellers draw their types independently from the uniform distribution over the set $[0,1]$, then the above results simplify to the following ones:

$$
\begin{aligned}
s^{*}\left(\theta_{i}\right) & =\theta_{i}+\frac{\left(1-\theta_{i}\right)}{n} \\
U_{i}\left(1 \mid \theta_{i}\right) & =\frac{\left(1-\theta_{i}\right)^{n}}{n} \\
R_{i}\left(1 \mid \theta_{i}\right) & =\theta_{i}\left(1-\theta_{i}\right)^{n-1}+\frac{\left(1-\theta_{i}\right)^{n}}{n} \\
U_{b}\left(1 \mid \theta_{b}\right) & =\theta_{b}-\frac{2}{n+1} \\
R_{b}\left(1 \mid \theta_{b}\right) & =\frac{2}{n+1} .
\end{aligned}
$$

Proof of Part 1: Seller's Optimal Response Strategy: Let us assume that after learning her type $\theta_{b}$, the buyer takes an action $\hat{\theta_{b}}$, which is essentially the maximum amount she is willing to pay. For any such reserve price announced by the buyer, we wish to compute the set $R\left(\hat{\theta}_{b}\right)$ - the optimal response (or rational reaction) set of the sellers. Recall from Section II-C, that the set $R\left(\hat{\theta}_{b}\right)$ essentially consists of all the pure strategy profiles of the sellers that are pure strategy Bayesian Nash equilibria of the induced Bayesian game among the sellers due to the action of the buyer. Let us assume that

$$
\begin{aligned}
R\left(\hat{\theta}_{b}\right)= & \left\{\left(s_{1}^{*}(\cdot), \ldots, s_{n}^{*}(\cdot)\right) \mid s_{i}^{*}:[\underline{\theta}, \bar{\theta}] \Rightarrow[\underline{\theta}, \bar{\theta}],\right. \\
& \left.\left(s_{1}^{*}(\cdot), \ldots, s_{n}^{*}(\cdot)\right) \text { is a Bayesian Nash equilibrium }\right\} .
\end{aligned}
$$

A considerable amount of investigation has been made for the past two decades in order to understand the structure of the set $R\left(\hat{\theta_{b}}\right)$ —see [14], [15], [17], [18], [13], and [19]-[21]. Recently,
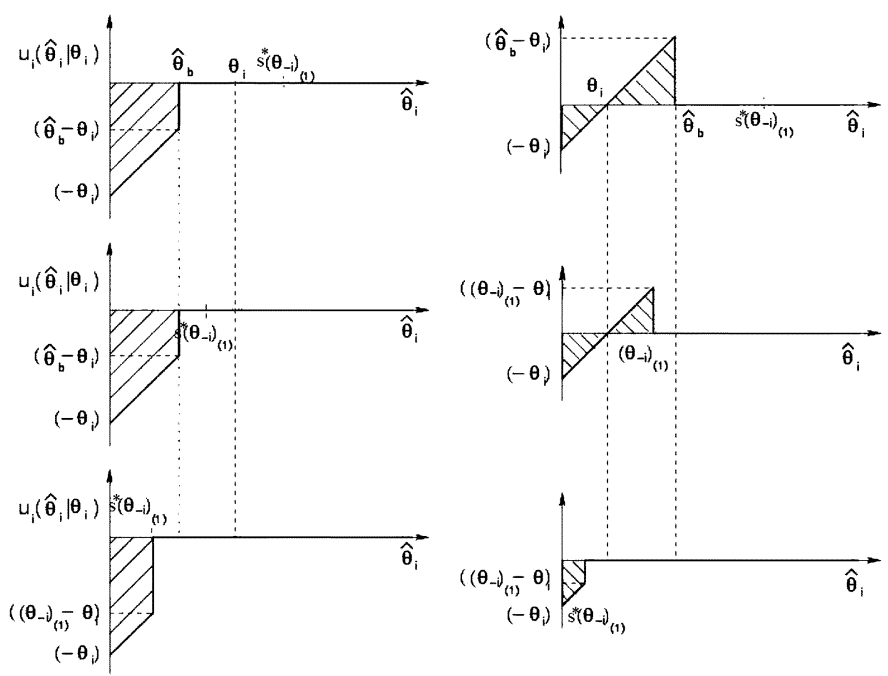

Fig. 1. The payoff of a seller in first-price procurement auction with reserve prices.

Maskin and Riley [17] have shown that under the assumptions A1-A4, the following holds true.

- The set $R\left(\hat{\theta_{b}}\right)$ is a singleton set.

- The optimal strategies of the sellers, that is $s_{i}^{*}(\cdot)$, are the same for all the sellers. With slight abuse of notation, we denote the equilibrium strategy of each agent $i$ by $s^{*}(\cdot)$ in which case we have $R\left(\hat{\theta_{b}}\right)=\left\{\left(s^{*}(\cdot), \ldots, s^{*}(\cdot)\right)\right\} \forall \hat{\theta_{b}} \in$ $[\underline{\theta}, \bar{\theta}]$.

- The optimal strategy $s^{*}(\cdot)$ of each seller is a strictly increasing and differentiable function.

Maskin and Riley [17] have shown these results for the first price selling auction with reserve prices, the same results can trivially be extended to the case of first price procurement auction with reserve prices. Using these results, we now proceed towards deriving the exact formula for $s^{*}(\cdot)$.

Fig. 1 shows the plot of how the payoff of a seller $i$, (denoted by $u_{i}\left(\hat{\theta}_{i} \mid \theta_{i}\right)$ ) varies if he reports his type to be $\hat{\theta}_{i}$, whereas his actual type (actual valuation for the object) is $\theta_{i}$. Notice that the payoff of seller $i$ depends on reserve price $\hat{\theta}_{b}$ announced by the buyer and the lowest reported type among the rest of the sellers. Since $s^{*}(\cdot)$ is a strictly increasing function, the ordering of the reported types $s^{*}\left(\theta_{1}\right), \ldots, s^{*}\left(\theta_{n}\right)$ will be the same as the ordering of the true types of the sellers. Therefore, the lowest reported type among the rest of the sellers would be $s^{*}\left(\left(\theta_{-i}\right)_{(1)}\right)$. The left-hand side curves in Fig. 1 depict the scenario when $\hat{\theta}_{b} \leq \theta_{i}$, whereas the right-hand side curves depict the scenario when $\theta_{i} \leq \hat{\theta}_{b}$. The three curves on either side discriminate the cases based on the value of the lowest reported type among rest of the sellers. The observations below follow trivially from these curves.

Observation 4.1: If $\theta_{i} \in\left[\hat{\theta_{b}}, \bar{\theta}\right]$, then for any seller $i$

$$
\left[u_{i}\left(\hat{\theta}_{i} \mid \theta_{i}\right) \leq u_{i}\left(\theta_{i} \mid \theta_{i}\right), \forall \hat{\theta}_{i} \in[\underline{\theta}, \bar{\theta}] \forall\left(\theta_{-i}\right)_{(1)} \in[\underline{\theta}, \bar{\theta}] .\right.
$$

Observation 4.2: If $\theta_{i} \in\left[\underline{\theta}, \hat{\theta_{b}}\right]$, then for any seller $i$

$$
\begin{aligned}
& u_{i}\left(\hat{\theta}_{i} \mid \theta_{i}\right) \leq u_{i}\left(\theta_{i} \mid \theta_{i}\right), \\
& \forall \hat{\theta}_{i} \in\left[\underline{\theta}, \theta_{i}\right], \forall \hat{\theta}_{i} \in\left(\hat{\theta}_{b}, \bar{\theta}\right] \forall\left(\theta_{-i}\right)_{(1)} \in[\underline{\theta}, \bar{\theta}] .
\end{aligned}
$$


Note that Observation 4.1 immediately implies the first part of the function $s^{*}\left(\theta_{i}\right)$. For the second part, note that Observation 4.2 implies that

1) $s^{*}\left(\theta_{i}\right)$ must lie in the interval $\left[\theta_{i}, \hat{\theta_{b}}\right]$;

2) $s^{*}\left(\hat{\theta}_{b}\right)=\hat{\theta}_{b}$.

In order to compute $s^{*}\left(\theta_{i}\right)$ for this scenario, we would first compute the expected gain of the seller $i$, denoted by $U_{i}\left(\hat{\theta}_{i} \mid \theta_{i}\right)$, if he reports his type to be $\hat{\theta}_{i}$, while his actual type is $\theta_{i}$ under the case when $\theta_{i} \in\left[\underline{\theta}, \hat{\theta_{b}}\right]$. Remember that at the same time all the other sellers are also reporting their types according to the strategy $s^{*}(\cdot)$. The following expression is a direct consequence of the right-hand side curves in the Fig. 16:

$$
\begin{aligned}
& U_{i}\left(\hat{\theta}_{i} \mid \theta_{i}\right)=\left[P\left(s^{*}\left(\left(\theta_{-i}\right)_{(1)}\right) \geq \hat{\theta_{b}}\right)\right. \\
& \left.\quad+P\left(\hat{\theta}_{b} \geq s^{*}\left(\left(\theta_{-i}\right)_{(1)}\right) \geq \hat{\theta}_{i}\right)\right]\left(\hat{\theta}_{i}-\theta_{i}\right) \\
& =\left[1-\Phi\left(s^{*^{-1}}\left(\hat{\theta_{b}}\right)\right)\right]^{n-1}\left(\hat{\theta}_{i}-\theta_{i}\right) \\
& \quad+\int_{s^{*-1}\left(\hat{\theta}_{i}\right)}^{s^{*-1}\left(\hat{\theta}_{b}\right)} \phi_{\left(\theta_{-i}\right)_{(1)}}(x)\left(\hat{\theta}_{i}-\theta_{i}\right) d x \\
& =\left[1-\Phi\left(s^{*^{-1}}\left(\hat{\theta}_{i}\right)\right)\right]^{n-1}\left(\hat{\theta}_{i}-\theta_{i}\right) .
\end{aligned}
$$

In order to compute an optimal strategy for seller $i$, we must apply the first-order necessary condition, which would imply that

$$
\begin{aligned}
& \frac{d U_{i}\left(\hat{\theta}_{i} \mid \theta_{i}\right)}{d \hat{\theta}_{i}}=0 \\
& \Rightarrow \quad\left[1-\Phi\left(s^{*^{-1}}\left(\hat{\theta}_{i}\right)\right)\right]^{n-1}+\left\{\left(\hat{\theta}_{i}-\theta_{i}\right)(n-1)\right. \\
& \\
& {\left.\left[1-\Phi\left(s^{*^{-1}}\left(\hat{\theta}_{i}\right)\right)\right]^{n-2}\left(-\phi\left({s^{*}}^{-1}\left(\hat{\theta}_{i}\right)\right)\right)\left(s^{*^{-1^{\prime}}}\left(\hat{\theta}_{i}\right)\right)\right\}=0 . }
\end{aligned}
$$

We know that the function $s^{*}(\cdot)$ which results from the above relation is an optimal strategy for the seller $i$. Therefore, it must also satisfy that $s^{*^{-1}}\left(\hat{\theta}_{i}\right)=\theta_{i}$. This fact would transform the above relation into the following first-order differential equation:

$$
s^{*^{\prime}}\left(\theta_{i}\right)=\left(s^{*}\left(\theta_{i}\right)-\theta_{i}\right)(n-1) \phi\left(\theta_{i}\right) /\left[1-\Phi\left(\theta_{i}\right)\right] .
$$

It is straightforward to show that the solution of the above o.d.e. would be

$$
s^{*}\left(\theta_{i}\right)=\theta_{i}+\frac{1}{\left[1-\Phi\left(\theta_{i}\right)\right]^{n-1}} \int_{\theta_{i}}^{\hat{\theta_{b}}}[1-\Phi(x)]^{n-1} d x .
$$

Buyer's Secure Strategy: We have shown that for any action $\hat{\theta_{b}}$ taken by the buyer, there is only one strategy profile with which the sellers will respond. Thus, in order to find out the secure strategy for the buyer, we must compute the expected payoff of the buyer when she announces a reserve price of $\hat{\theta}_{b}$, while her actual type is $\theta_{b}$. Notice that due to the monotonicity of the function $s^{*}(\cdot)$, the minimum reported type is given by $s^{*}\left(\theta_{(1)}\right)$. It is easy to see that if $\hat{\theta}_{b}<s^{*}\left(\theta_{(1)}\right)$ then $U_{b}\left(\hat{\theta}_{b} \mid \theta_{b}\right)=$

${ }^{6}$ In this expression, we have made use of the fact that $s^{*}(\cdot)$ is a strictly increasing, hence invertible, and differentiable function. We have also made use of some results about the CDF of order statistics of independent random variables [23].
0 otherwise $U_{b}\left(\hat{\theta_{b}} \mid \theta_{b}\right)=\left(\theta_{b}-s^{*}\left(\theta_{(1)}\right)\right)$. Thus, we can conclude that

$$
U_{b}\left(\hat{\theta_{b}} \mid \theta_{b}\right)=\int_{\underline{\theta}}^{\hat{\theta_{b}}} \phi_{\Theta_{(1)}}(x)\left(\theta_{b}-s^{*}(x)\right) d x .
$$

Substituting the value of $s^{*}(x)$, the above relation becomes

$$
\begin{aligned}
U_{b}\left(\hat{\theta_{b}} \mid \theta_{b}\right)=\theta_{b} \Phi_{\theta_{(1)}}\left(\hat{\theta_{b}}\right) & -\int_{\underline{\theta}}^{\hat{\theta_{b}}} x \phi_{\theta_{(1)}}(x) d x \\
& -\int_{\underline{\theta}}^{\hat{\theta_{b}}} n \phi(x)\left(\int_{x}^{\hat{\theta}_{b}}[1-\Phi(y)]^{n-1} d y\right) d x .
\end{aligned}
$$

By change of variables in double integration, we get

$$
\begin{aligned}
U_{b}\left(\hat{\theta_{b}} \mid \theta_{b}\right)= & \theta_{b} \Phi_{\theta_{(1)}}\left(\hat{\theta_{b}}\right)-\int_{\underline{\theta}}^{\hat{\theta_{b}}} x \phi_{\theta_{(1)}}(x) d x \\
& -\int_{\underline{\theta}}^{\hat{\theta_{b}}}[1-\Phi(y)]^{n-1}\left(\int_{\underline{\theta}}^{y} n \phi(x) d x\right) d y \\
= & \theta_{b} \Phi_{\theta_{(1)}}\left(\hat{\theta_{b}}\right)-\int_{\underline{\theta}}^{\hat{\theta_{b}}} x \phi_{\theta_{(1)}}(x) d x \\
& -n \int_{\underline{\theta}}^{\hat{\theta_{b}}} \Phi(y)[1-\Phi(y)]^{n-1} d y \\
= & \left(\theta_{b}-\hat{\theta_{b}}\right) \Phi_{\Theta_{(1)}}\left(\hat{\theta_{b}}\right)+\int_{x=\underline{\theta}}^{\hat{\theta_{b}}}\left[\Phi_{\Theta_{(2)}}(x)\right] d x .
\end{aligned}
$$

In order to compute an optimal reserve pricing strategy for the buyer, we must apply the first order necessary condition, which would imply that

$$
\begin{aligned}
& \frac{d U_{b}\left(\hat{\theta_{b}} \mid \theta_{b}\right)}{d \hat{\theta_{b}}=0} \\
& \Rightarrow \quad\left(\theta_{b}-\hat{\theta_{b}}\right) \phi_{\Theta_{(1)}}\left(\hat{\theta_{b}}\right)-\Phi_{\Theta_{(1)}}\left(\hat{\theta_{b}}\right)+\Phi_{\Theta_{(2)}}\left(\hat{\theta_{b}}\right)=0 .
\end{aligned}
$$

Substituting the values of $\phi_{\Theta_{(1)}}\left(\hat{\theta_{b}}\right), \Phi_{\Theta_{(1)}}\left(\hat{\theta_{b}}\right)$, and $\Phi_{\Theta_{(2)}}\left(\hat{\theta_{b}}\right)$ from the appendix, we will get

$$
\theta_{b}=\hat{\theta_{b}}+\frac{\Phi\left(\hat{\theta_{b}}\right)}{\phi\left(\hat{\theta_{b}}\right)} .
$$

Replacing $\hat{\theta_{b}}$ with $s_{b}^{*}\left(\theta_{b}\right)$, we get the following expression:

$$
\theta_{b}=s_{b}^{*}\left(\theta_{b}\right)+\frac{\Phi\left(s_{b}^{*}\left(\theta_{b}\right)\right)}{\phi\left(s_{b}^{*}\left(\theta_{b}\right)\right)} .
$$

Proof of Part 2: We have already shown in Part 1 that for any announced reserve price $\hat{\theta_{b}}$, a seller will report $s^{*}\left(\theta_{i}\right)$ as his type if his actual type is $\theta_{i}$. It was also shown that $s^{*}\left(\theta_{i}\right)=$ $\theta_{i} \forall \theta_{i} \in\left[\hat{\theta}_{b}, \bar{\theta}\right]$ which essentially implies that this seller cannot 
be the winner and hence his expected payoff $U\left(\hat{\theta_{b}} \mid \theta_{i}\right)$, as well as the expected revenue received $R\left(\hat{\theta}_{b} \mid \theta_{i}\right)$ will be zero. This proves the first part of the function definition $U\left(\hat{\theta}_{b} \mid \theta_{i}\right)$ and $R\left(\hat{\theta_{b}} \mid \theta_{i}\right)$.

In order to get the second part, we just need to replace $\hat{\theta}_{i}$ in (38) by $s^{*}\left(\theta_{i}\right)$ because in equilibrium each seller will report a type according to the strategy $s^{*}\left(\theta_{i}\right)$. This implies that

$$
\begin{aligned}
U_{i}\left(\hat{\theta_{b}} \mid \theta_{i}\right) & =U_{i}\left(s^{*}\left(\theta_{i}\right) \mid \theta_{i}\right) \\
& =\left[1-\Phi\left(s^{*^{-1}}\left(s^{*}\left(\theta_{i}\right)\right)\right)\right]^{n-1}\left(s^{*}\left(\theta_{i}\right)-\theta_{i}\right) \\
& =\left[1-\Phi\left(\theta_{i}\right)\right]^{n-1}\left(s^{*}\left(\theta_{i}\right)-\theta_{i}\right) \\
& =\int_{\theta_{i}}^{\theta_{b}}[1-\Phi(x)]^{n-1} d x .
\end{aligned}
$$

In order to get $R\left(\hat{\theta}_{b} \mid \theta_{i}\right)$, we observe that the probability of seller $i$ being the winner is equal to the probability that $s^{*}\left(\theta_{i}\right)$ is the smallest reported type among all the reported types which, in turn, is the same as the probability that $\theta_{i}$ is the minimum among all the types of all the sellers. This turns out to be $\left(1-\Phi\left(\theta_{i}\right)\right)^{n-1}$. Thus, the expected value loss to the seller is $\theta_{i}\left(1-\Phi\left(\theta_{i}\right)\right)^{n-1}$ and it is easy to see that expected revenue received by the seller is equal to the expected values loss incurred to him plus expected payoff.

Proof of Part 3: The expression for $U_{b}\left(\hat{\theta_{b}} \mid \theta_{b}\right)$ directly follows from (41) and the expression for $R_{b}\left(\hat{\theta_{b}} \mid \theta_{b}\right)$ can be derived in a similar fashion as we derived the expression for $R_{i}\left(\hat{\theta}_{b} \mid \theta_{i}\right)$ in Part 2. (Q.E.D.).

Summary of Theorem 4.1: In this theorem, we have shown that F-PAR is neither BaSIC for the buyer nor for the seller. This is because, for each agent, his or her true type is not an optimal announcement. We have derived the equilibrium bidding strategies for both the buyer and the sellers. We have also derived formulae for the expected utility and the expected payment received by both the buyer and the sellers. The equilibrium bidding strategies were developed by first computing the seller's optimal response strategy against any strategy followed by the buyer, and then computing the buyer's secure strategy which translates into her equilibrium strategy. The corresponding optimal response strategy of a seller becomes his equilibrium strategy. The computation of utility and payment for the buyer and the sellers are fairly straightforward because of the way we derive the equilibrium strategies.

Theorem 4.2: Under the assumptions A1-A4,

1) The SCF used in the second-price procurement auction with reserve prices, which is given by (13), (14), and (17) is $\mathrm{BaSIC}$ for the followers but is not BaSIC for the leader. The Bayesian Stackelberg equilibrium of the Bayesian Stackelberg game induced by this function among the sellers and the buyer is given by $s^{*}=\left(s_{b}^{*}, t_{-b}^{*}\right)$, where

1.1) $s_{b}^{*}(\cdot)$ is the solution of the following equation:

$$
\theta_{b}=s_{b}^{*}\left(\theta_{b}\right)+\frac{\Phi\left(s_{b}^{*}\left(\theta_{b}\right)\right)}{\phi\left(s_{b}^{*}\left(\theta_{b}\right)\right)} .
$$

1.2) $t_{-b}^{*}\left(\hat{\theta_{b}}\right)=\left(s^{*}(\cdot), \ldots, s^{*}(\cdot)\right) \forall \hat{\theta_{b}} \in \Theta_{b}=[\underline{\theta}, \bar{\theta}]$, where

$$
s^{*}\left(\theta_{i}\right)=\theta_{i} \forall \theta_{i} \in \Theta_{i}=[\underline{\theta}, \bar{\theta}] .
$$
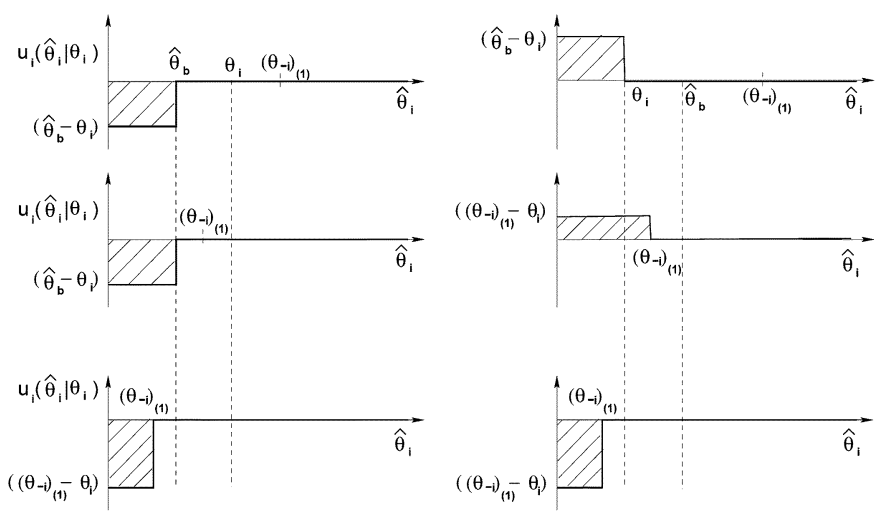

Fig. 2. The payoff of a seller in second-price procurement auction with reserve prices.

That is, buyer always announces $s_{b}^{*}\left(\theta_{b}\right)$ as the reserve price if her true valuation is $\theta_{b}$. For any reserve price $\hat{\theta}_{b}$ announced by the buyer, the sellers always report their true valuation (type).

2) For an announced reserve price of $\hat{\theta}_{b}$ by the buyer, the expected payoff (utility) $U_{i}\left(\hat{\theta}_{b} \mid \theta_{i}\right)$ and the expected revenue $R_{i}\left(\hat{\theta}_{b} \mid \theta_{i}\right)$ received by any seller $i$, when his actual type is $\theta_{i}$, is given by the following expressions:

$$
\begin{aligned}
& U_{i}\left(\hat{\theta_{b}} \mid \theta_{i}\right)= \begin{cases}0, & \theta_{i} \in\left[\hat{\hat{\theta}_{b}}, \bar{\theta}\right] \\
\int_{\theta_{i}}(1-\Phi(x))^{n-1} d x, & \theta_{i} \in\left[\underline{\theta}, \hat{\theta_{b}}\right]\end{cases} \\
& R_{i}\left(\hat{\theta_{b}} \mid \theta_{i}\right)=\left\{\begin{array}{ll}
0, & \theta_{i} \in\left[\hat{\theta_{b}}, \overline{\theta^{\prime}}\right] \\
\left\{\theta_{i}\left(1-\Phi\left(\theta_{i}\right)\right)^{n-1}+,\right. & \theta_{i} \in\left[\underline{\theta}, \hat{\theta_{b}}\right] \\
\left.U_{i}\left(\hat{\theta_{b}} \mid \theta_{i}\right)\right\}, &
\end{array} .\right.
\end{aligned}
$$

3) When actual type of the buyer is $\theta_{b}$ and she announces a reserve price $\hat{\theta_{b}}$, her expected payoff (utility) $U_{b}\left(\hat{\theta_{b}} \mid \theta_{b}\right)$ and the expected payment $R_{b}\left(\hat{\theta_{b}} \mid \theta_{b}\right)$ made by her turn out to be the following:

$$
\begin{aligned}
& U_{b}\left(\hat{\theta_{b}} \mid \theta_{b}\right)=\left(\theta_{b}-\hat{\theta_{b}}\right) \Phi_{\Theta_{(1)}}\left(\hat{\theta_{b}}\right)+\int_{x=\underline{\theta}}^{\hat{\theta_{b}}}\left[\Phi_{\Theta_{(2)}}(x)\right] d x \\
& R_{b}\left(\hat{\theta_{b}} \mid \theta_{b}\right)=\theta_{b} \Phi_{\Theta_{(1)}}\left(\hat{\theta_{b}}\right)-U_{b}\left(\hat{\theta_{b}} \mid \theta_{b}\right) .
\end{aligned}
$$

Corollary 4.2:

1) The optimal reserve price strategy of the buyer in S-PAR when all the sellers draw their types independently from uniform distribution over the set $[0,1]$ is given by $s_{b}^{*}\left(\theta_{b}\right)=$ $\theta_{b} / 2$.

Proof of Part 1: Seller's Optimal Response Strategy: As before, we start with computing the optimal response (or rational reaction) set of the sellers $R\left(\hat{\theta_{b}}\right)$ against any reserve price $\hat{\theta_{b}}$ announced by the buyer. Fig. 2 is the analog of Fig. 1 for S-PAR. The following two observations are a direct consequence of this figure.

Observation 4.3: If $\theta_{i} \in\left[\hat{\theta_{b}}, \bar{\theta}\right]$, then for seller $i$

$$
u_{i}\left(\hat{\theta}_{i} \mid \theta_{i}\right) \leq u_{i}\left(\theta_{i} \mid \theta_{i}\right) \forall \hat{\theta}_{i} \in[\underline{\theta}, \bar{\theta}] \forall\left(\theta_{-i}\right)_{(1)} \in[\underline{\theta}, \bar{\theta}]
$$

Observation 4.4: If $\theta_{i} \in\left[\underline{\theta}, \hat{\theta_{b}}\right]$, then for seller $i$

$$
u_{i}\left(\hat{\theta}_{i} \mid \theta_{i}\right) \leq u_{i}\left(\theta_{i} \mid \theta_{i}\right), \forall \hat{\theta}_{i} \in[\underline{\theta}, \bar{\theta}] \forall\left(\theta_{-i}\right)_{(1)} \in[\underline{\theta}, \bar{\theta}] .
$$




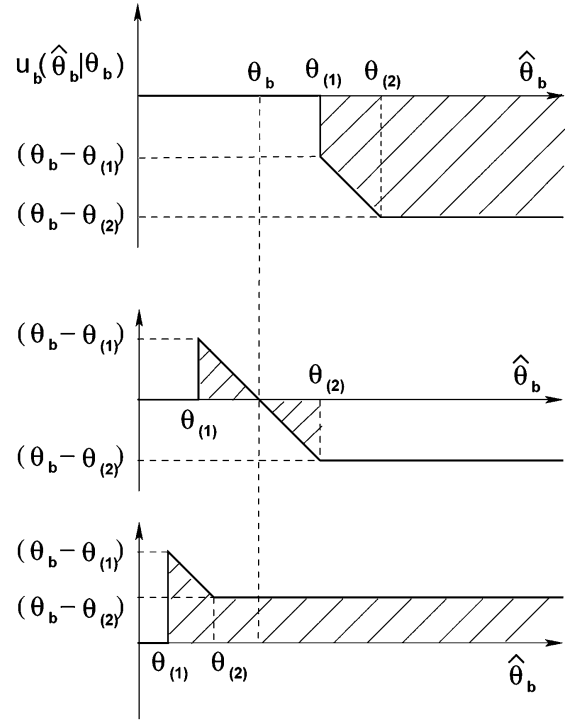

Fig. 3. The payoff of buyer in second-price procurement auction with reserve prices.

These two observations immediately imply that truth revelation is a weakly dominant strategy for each seller. Therefore, we have

$$
R\left(\hat{\theta_{b}}\right)=\left\{\left(s^{*}(\cdot), \ldots, s^{*}(\cdot)\right) \mid s^{*}\left(\theta_{i}\right)=\theta_{i} \forall \theta_{i} \in \Theta_{i}=[\underline{\theta}, \bar{\theta}]\right\} .
$$

This proves the first part of the theorem that the SCF is BaSIC for the followers. To prove the next part, we need to compute the buyer's secure strategy.

Buyer's Secure Strategy: We have shown that irrespective of what reserve price is announced by the buyer, it is always a weakly dominant strategy for the sellers to report their true valuation about the object. Therefore, the lowest reported type $\theta_{(1)}$ and the second lowest reported type $\theta_{(2)}$ are independent of the value of reserve price $\hat{\theta}_{b}$. Based on this argument, we have drawn Fig. 3, which depicts the payoff to the buyer when she reports her type to be $\hat{\theta}_{b}$, while her actual type is $\theta_{b}$. We denote this payoff by $U_{b}\left(\hat{\theta}_{b} \mid \theta_{b}\right)$. The figure consists of three curves each for a different possible combination of the values of $\theta_{(1)}$ and $\theta_{(2)}$. The following observation is a direct consequence of this figure.

Observation 4.5: $U_{b}\left(\hat{\theta_{b}} \mid \theta_{b}\right) \leq U_{b}\left(\theta_{b} \mid \theta_{b}\right), \forall \hat{\theta_{b}} \in\left[\theta_{b}, \bar{\theta}\right]$.

This observation implies that $s_{b}^{*}\left(\theta_{b}\right) \in\left[\underline{\theta}, \theta_{b}\right] \forall \theta_{b} \in[\underline{\theta}, \bar{\theta}]$. Thus, we have a boundary condition $s_{b}^{*}(\underline{\theta})=\underline{\theta}$. The expression for expected payoff $U_{b}\left(\hat{\theta_{b}} \mid \theta_{b}\right)$ of the buyer when she announces a reserve price of $\hat{\theta_{b}} \in\left[\underline{\theta}, \theta_{b}\right]$, while her actual type is $\theta_{b}$, follows immediately from Fig. 3 :

$$
\begin{aligned}
& U_{b}\left(\hat{\theta_{b}} \mid \theta_{b}\right) \\
&= P\left(\underline{\theta} \leq \theta_{(1)} \leq \hat{\theta_{b}}, \hat{\theta_{b}} \leq \theta_{(2)} \leq \bar{\theta}\right)\left(\theta_{b}-\hat{\theta_{b}}\right) \\
&+P\left(\underline{\theta} \leq \theta_{(2)} \leq \hat{\theta_{b}}\right)\left(\theta_{b}-\theta_{(2)}\right) \\
&=\left(\Phi_{\left(\Theta_{(1)}, \Theta_{(2)}\right)}\left(\hat{\theta_{b}}, \bar{\theta}\right)-\Phi_{\left(\Theta_{(1)}, \Theta_{(2)}\right)}(\underline{\theta}, \bar{\theta})\right. \\
&\left.-\Phi_{\left(\Theta_{(1)}, \Theta_{(2)}\right)}\left(\hat{\theta_{b}}, \hat{\theta_{b}}\right)+\Phi_{\left(\Theta_{(1)}, \Theta_{(2)}\right)}\left(\underline{\theta}, \hat{\theta_{b}}\right)\right)\left(\theta_{b}-\hat{\theta}_{b}\right) \\
&+\int_{x=\underline{\theta}}^{\hat{\theta_{b}}} \phi_{\Theta_{(2)}}(x)\left(\theta_{b}-x\right) d x
\end{aligned}
$$

where $\Phi_{\left(\Theta_{(1)}, \Theta_{(2)}\right)}(\ldots)$ is a joint CDF of the first-order and second-order statistics $\Theta_{(1)}$ and $\Theta_{(2)} . \Phi_{\Theta_{(1)}}(\cdot)$ and $\Phi_{\Theta_{(2)}}(\cdot)$ are the marginal CDF of the $\Theta_{(1)}$ and $\Theta_{(2)}$, respectively. Similarly, $\phi_{\Theta_{(2)}}(\cdot)$ is the marginal PDF of $\Theta_{(2)}$. The formulae for these quantities are summarized in the appendix. Making use of the relations given in the appendix, (42) can be written as

$$
\begin{aligned}
U_{b}\left(\hat{\theta_{b}} \mid \theta_{b}\right)=n \Phi\left(\hat{\theta_{b}}\right)\left[1-\Phi\left(\hat{\theta_{b}}\right)\right]^{n-1}\left(\theta_{b}-\hat{\theta_{b}}\right) & \\
& +\int_{x=\underline{\theta}}^{\theta_{b}} \phi_{\Theta_{(2)}}(x)\left(\theta_{b}-x\right) d x .
\end{aligned}
$$

Integrating by parts, we will obtain the following relation:

$$
\begin{aligned}
U_{b}\left(\hat{\theta_{b}} \mid \theta_{b}\right) \\
=n \Phi\left(\hat{\theta_{b}}\right)\left[1-\Phi\left(\hat{\theta_{b}}\right)\right]^{n-1}\left(\theta_{b}-\hat{\theta_{b}}\right) \\
\quad+\left|\left(\theta_{b}-x\right) \Phi_{\Theta_{(2)}}(x)\right|_{x=\underline{\theta}}^{\hat{\theta_{b}}}+\int_{x=\underline{\theta}}^{\hat{\theta_{b}}} \Phi_{\Theta_{(2)}}(x) d x \\
=\left(\theta_{b}-\hat{\theta_{b}}\right)\left[1-\left(1-\Phi\left(\hat{\theta_{b}}\right)\right)^{n}\right] \\
\quad+\int_{x=\underline{\theta}}^{\hat{\theta_{b}}} \Phi_{\Theta_{(2)}}(x) d x=\left(\theta_{b}-\hat{\theta_{b}}\right) \Phi_{\Theta_{(1)}}\left(\hat{\theta_{b}}\right) \\
\quad+\int_{x=\underline{\theta}}^{\hat{\theta_{b}}} \Phi_{\Theta_{(2)}}(x) d x .
\end{aligned}
$$

Note that for a given type $\theta_{b}$ of the buyer, the expected utility function $U_{b}\left(\hat{\theta_{b}} \mid \theta_{b}\right)$ is a mapping from $[\underline{\theta}, \bar{\theta}]$ into $\mathbb{R}$. It is easy to verify that $U_{b}\left(\hat{\theta_{b}} \mid \theta_{b}\right)$ is a continuous mapping over a compact interval and, therefore, due to the Weierstrass theorem, it must attain a maximum and a minimum at some points in the interval $[\underline{\theta}, \bar{\theta}]$. The point of maximum will be the optimal reserve price that the buyer should announce if her type is $\theta_{b}$. In order to compute an optimal reserve pricing strategy for the buyer, we must apply the first-order necessary condition, which would imply that

$$
\begin{aligned}
& \frac{d U_{b}\left(\hat{\theta_{b}} \mid \theta_{b}\right)}{d \hat{\theta_{b}}}=0 \\
& \quad \Rightarrow \quad\left(\theta_{b}-\hat{\theta_{b}}\right) \phi_{\Theta_{(1)}}\left(\hat{\theta_{b}}\right)-\Phi_{\Theta_{(1)}}\left(\hat{\theta_{b}}\right)+\Phi_{\Theta_{(2)}}\left(\hat{\theta_{b}}\right)=0 .
\end{aligned}
$$

Substituting the values of $\phi_{\Theta_{(1)}}\left(\hat{\theta_{b}}\right), \Phi_{\Theta_{(1)}}\left(\hat{\theta_{b}}\right)$, and $\Phi_{\Theta_{(2)}}\left(\hat{\theta_{b}}\right)$ from the appendix, we will get

$$
\theta_{b}=\hat{\theta_{b}}+\frac{\Phi\left(\hat{\theta_{b}}\right)}{\phi\left(\hat{\theta_{b}}\right) .}
$$

Replacing $\hat{\theta_{b}}$ with $s_{b}^{*}\left(\theta_{b}\right)$, we get the following expression:

$$
\theta_{b}=s_{b}^{*}\left(\theta_{b}\right)+\frac{\Phi\left(s_{b}^{*}\left(\theta_{b}\right)\right)}{\phi\left(s_{b}^{*}\left(\theta_{b}\right)\right)} .
$$

This proves Part 1 of the theorem.

Proof of Part 2: We have already shown in Part 1 that for any announced reserve price $\hat{\theta}_{b}$, a seller will always report his true type $\theta_{i}$. Therefore, this seller cannot be the winner if $\theta_{i} \in$ $\left[\hat{\theta}_{b}, \bar{\theta}\right]$ in which case his expected payoff $U_{i}\left(\hat{\theta}_{b} \mid \theta_{i}\right)$, as well as the expected revenue received $R_{i}\left(\hat{\theta}_{b} \mid \theta_{i}\right)$ will be zero. This proves 
the first part of the function definition $U_{i}\left(\hat{\theta_{b}} \mid \theta_{i}\right)$ and $R_{i}\left(\hat{\theta_{b}} \mid \theta_{i}\right)$. For the second part, we can make use of Fig. 2 and write that

$$
\begin{aligned}
U_{i}\left(\hat{\theta_{b}} \mid \theta_{i}\right)=\left(\left(\theta_{-i}\right)_{(1)}-\theta_{i}\right)\left[P\left(\theta_{i} \leq\left(\theta_{-i}\right)_{(1)} \leq \hat{\theta_{b}}\right)\right] \\
\quad+\left(\hat{\theta_{b}}-\theta_{i}\right)\left[P\left(\hat{\theta}_{b} \leq\left(\theta_{-i}\right)_{(1)}\right)\right] \\
=\int_{\theta_{i}}^{\hat{\theta}_{b}} \phi_{\Theta_{-i(1)}}(x)\left(x-\theta_{i}\right) d x \\
\quad+\left(\hat{\theta_{b}}-\theta_{i}\right)\left[1-\Phi_{\Theta_{-i}(1)}\left(\hat{\theta_{b}}\right)\right] \\
=\int_{\theta_{i}}^{\hat{\theta_{b}}}\left(1-\Phi_{\Theta_{-i(1)}}(x)\right) d x \\
=\int_{\theta_{i}}^{\theta_{b}}(1-\Phi(x))^{n-1} d x .
\end{aligned}
$$

The expression for $R_{i}\left(\hat{\theta}_{b} \mid \theta_{i}\right)$ can be obtained in a similar manner as we did it in the proof of Part 2 of Theorem 4.1.

Proof of Part 3: The expression for $U_{b}\left(\hat{\theta}_{b} \mid \theta_{b}\right)$ directly follows from (43) and the expression for $R_{b}\left(\hat{\theta_{b}} \mid \theta_{b}\right)$ can be derived in a similar fashion as we derived the expression for $R_{i}\left(\theta_{b} \mid \theta_{i}\right)$ in Theorem 4.1. (Q.E.D.).

Following are a few important observations concerning Theorem 4.1 and Theorem 4.2.

1) For an announced reserve price $\hat{\theta}_{b}$, the expected utility $U_{i}\left(\hat{\theta_{b}} \mid \theta_{i}\right)$ and expected payment $R_{i}\left(\hat{\theta_{b}} \mid \theta_{i}\right)$ received by a seller turn out to be the same for both F-PAR and S-PAR.

2) The optimal reserve price strategy of the buyer $s_{b}^{*}(\cdot)$ is the same for both F-PAR and S-PAR.

3) For an announced reserve price $\hat{\theta}_{b}$, the expected utility $U_{b}\left(\hat{\theta_{b}} \mid \theta_{b}\right)$ of the buyer and the expected payment $R_{b}\left(\hat{\theta_{b}} \mid \theta_{b}\right)$ made by the buyer to the winning seller turn out to be the same for both F-PAR and S-PAR. This confirms the classical Revenue Equivalence theorem [12], [24]-[28].

Summary of Theorem 4.2: In this theorem, we have shown that S-PAR is BaSIC for the sellers but not for the buyer. We have computed the equilibrium bidding strategies for the buyer as well as for the sellers. We have also derived formulae for the expected utility and the expected payment received by both the buyer and the sellers. The approach followed in this proof closely parallels that of Theorem 4.1 .

\section{SUMMARY AND FUTURE WORK}

In this paper, we have extended the classical mechanism design theory to the specific setting of single leader rest follower problems, which are a special class of hierarchical decision making problems. We have also explored the application of the theory developed to investigate incentive compatibility properties of the first-price and second-price procurement auctions with reserve prices. The framework developed in this paper can be immediately applied for modeling and solving mechanism design problems arising in many emerging applications with a two-level hierarchical structure. We have already provided a listing of these applications in Section I.

In this paper, our focus was on the problems which have only two levels within the hierarchy, with only one agent at the higher level. However, in practice, problems such as organizational decision making, decision making within the
Government sector, scheduling decisions in computational grids, and routing decisions within communication networks involve multiple levels within the hierarchy of decision makers. In order to address these problems, we need to advance the theory from SLRF mechanism design problems to general Stackelberg mechanism design problems. This is another promising direction for future work.

This paper has only addressed theoretical issues in the context of SLRF mechanism design. The actual design of SLRF auctions and examining the data of real-world auctions to design such auctions in a better way also need to be looked at.

\section{APPENDIX \\ ORDER STATISTICS}

Let $\Theta_{1}, \Theta_{2}, \ldots, \Theta_{n}$ be $n$ i.i.d. random variables of the continuous type with $\operatorname{CDF} \Phi(x)$ and $\operatorname{PDF} \phi(x)$. For any realization $\left(\theta_{1}, \ldots, \theta_{n}\right)$ of these random variables, we define $\theta_{(k)}$ as $k$ th smallest element in $\left(\theta_{1}, \ldots, \theta_{n}\right)$, which is also known as $k$ th order statistic. Thus, we can define a $k$ th order statistic random variable $\Theta_{(k)}, k=1, \ldots, n$ whose realization is given by $\theta_{(k)}$. The marginal as well as joint CDF and PDF of the first- and the second-order statistics $\Theta_{(1)}$ and $\Theta_{(2)}$ turn out to be the following [29], [23]:

$$
\begin{aligned}
& \Phi_{\Theta_{(1)}}(x)= 1-[1-\Phi(x)]^{n} \\
& \Phi_{\Theta_{(2)}}(x)= \Phi_{\Theta_{(1)}}(x)-n \Phi(x)[1-\Phi(x)]^{n-1} \\
& \Phi_{\left(\Theta_{(1)}, \Theta_{(2)}\right)}(x, y)= \begin{cases}\Phi_{\left(\Theta_{(1)}, \Theta_{(2)}\right.}(y, y), & y<x \\
1-[1-\Phi(x)]^{n}- & \\
n \Phi(x)[1-\Phi(y)]^{n-1}, & x \leq y\end{cases} \\
& \phi_{\Theta_{(1)}}(x)=n[1-\Phi(x)]^{n-1} \phi(x) \\
& \phi_{\Theta_{(2)}}(x)=n(n-1)[1-\Phi(x)]^{n-2}[\Phi(x)] \phi(x) \\
& \phi_{\left(\Theta_{(1)}, \Theta_{(2)}\right)}(x, y)=\left\{\begin{array}{ll}
0, & y<x \\
n(n-1)[1-\Phi(y)]^{n-2} & x \leq y \\
\phi(x) \phi(y)\}, &
\end{array} .\right.
\end{aligned}
$$

The marginal CDF and PDF of the highest order statistic, i.e., $\theta_{(n)}$ is given by the following equations:

$$
\begin{aligned}
\Phi_{\Theta_{(n)}}(x) & =[\Phi(x)]^{n} \\
\phi_{\Theta_{(n)}}(x) & =n[\Phi(x)]^{n-1} \phi(x) .
\end{aligned}
$$

\section{ACKNOWLEDGMENT}

The authors would like to thank Dr. S. Das, Program Manager, Communications and Networking, for the encouragement and support.

\section{REFERENCES}

[1] V. H. Stackelberg, Marketform und Gleichgewicht. Oxford, U.K.: Oxford Univ. Press, 1934, an English translation appeared in 1952 entitled The Theory of Market Economy.

[2] J. F. Kurose and K. W. Ross, Computer Networking: A Top-Down Approach Featuring the Internet. Delhi, India, 2003, Pearson Education.

[3] A. Mas-Colell, M. D. Whinston, and J. R. Green, Microeconomic Theory. New York: Oxford Univ. Press, 1995.

[4] R. B. Myerson, Game Theory: Analysis of Conflict. Cambridge, MA: Harvard Univ. Press, 1997.

[5] J. Cremer and M. Riordan, "A sequential solution to the public goods problem," Econometrica, vol. 53, no. 1, pp. 77-84, 1985. 
[6] T. S. Chang and P. B. Luh, "Derivation of necessary and sufficient conditions for single-stage stackelberg games via the inducible region concept," IEEE Trans. Automat. Contr., vol. 29, no. 1, pp. 63-66, 1984.

[7] T. C. P. B. Luh and T. Ning, "Three-level Stackelberg decision problems," IEEE Trans. Automat. Contr., vol. 29, no. 3, pp. 280-282, 1984.

[8] T. S. C. P. B. Luh and S. C. Chang, "Solutions and properties of multistage stackelberg games," Automatica, vol. 20, no. 2, pp. 251-256, 1984.

[9] K. Konrad and W. Leininger, "The generalized Stackelberg equilibrium of the all-pay auction with complete information," EconPapers Working Paper, Tech. Rep., 2005.

[10] T. Başar and G. J. Olsder, Dynamic Noncooperative Game Theory. New York: Academic, 1982.

[11] V. Krishna, Auction Theory. New York: Academic, 2002.

[12] R. B. Myerson, "Optimal auction design," Math. Operations Res., vol. 6, no. 1, pp. 58-73, Feb. 1981.

[13] E. Maskin and J. Riley, "Equilibrium in sealed high bid auctions," Review of Economic Studies, vol. 67, pp. 439-454, 2000.

[14] P. R. Milgrom and R. R. Weber, "A theory of auctions and competitive bidding," Econometrica, vol. 50, no. 5, pp. 1089-1122, Sep. 1982.

[15] P. R. McAfee and J. McMillan, "Auctions and bidding," J. Econ. Literature, vol. 25 , no. 2, pp. 699-738, Jun. 1987.

[16] E. Wolfstetter, Topics in Microeconomics. Cambridge, U.K.: Cambridge Univ. Press, 1999.

[17] E. Maskin and J. Riley, "Uniqueness of equilibrium in sealed high bid auctions," Games Econ. Behav., vol. 45, pp. 395-409, 2003.

[18] E. Maskin and J. Riley, "Asymmetric auctions," Rev. Economic Studies, vol. 67 , no. 3, pp. 413-438, 2000.

[19] H. Cai, J. Riley, and L. Ye, "Reserve price signaling," J. Economic Theory, 2007, to be published.

[20] T. Li, "Econometrics of first-price auctions with entry and binding reservation prices," J. Econometrics, vol. 126, no. 1, pp. 173-200, May 2005.

[21] P. R. McAfee, "Search mechanisms," J. Econ. Theory, vol. 44, no. 1, pp. 99-123, Feb. 1988.

[22] E. Maskin and J. Riley, "Optimal auctions with risk averse buyers," Econometrica, vol. 52, no. 6, pp. 1473-1518, 1984.

[23] K. S. Trivedi, Probability and Statistics with Reliability, Queuing, and Computer Science Applications. New Delhi, India: Prentice-Hall of India Private Limited, 1994.

[24] W. Vickrey, "Counterspeculation, auctions, and competitive sealed tenders," J. Finance, vol. 16, no. 1, pp. 8-37, Mar. 1961.

[25] A. Ortega-Reichert, "Models for Competitive Bidding Under Uncertainty," Ph.D. dissertation, Dept. Oper. Res., Stanford Univ., Stanford, CA, Jan. 1968.

[26] J. Holt and C. A. , "Uncertainty and the bidding for incentive contracts," Amer. Econ. Rev., vol. 69, no. 4, pp. 697-705, Sep. 1979.

[27] M. Harris and A. Raviv, "Allocation mechanisms and the design of auctions," Econometrica, vol. 49, no. 6, pp. 1477-99, Nov. 1981.
[28] J. G. Riley and W. F. Samuelson, "Optimal auctions," Amer. Econ. Rev., vol. 71, no. 3, pp. 381-92, Jun. 1981.

[29] V. Rohatgi and A. M. E. Saleh, An Introduction to Probability and Statistics. New York: Wiley, 1976.

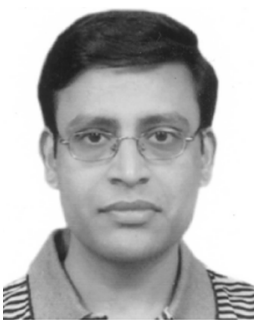

Dinesh Garg received the M.Sc. degree in engineering and the Ph.D. degree in computer science and automation from the Indian Institute of Science (IISc), Bangalore, in 2002 and 2006, respectively.

$\mathrm{He}$ is a Research Staff Member at IBM India Research Lab, Bangalore, India. His current research interests include game theory and mechanism design theory with applications to Online auctions, sponsored search auctions, and supply chain networks.

Dr. Garg has received the Annual Googol Best New Application Paper Award of the IEEE TRANSACTIONS ON AUTOMATION SCIENCE AND ENGINEERING for 2004. He is also a recipient of the Prof. F. N. Mowdawala Medal for the Best M.Sc. (Engineering) Thesis in the Division of Electrical Sciences at IISc, 2002-2003.

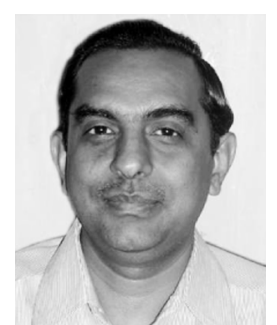

Y. Narahari (F'07) received the Ph.D. degree from the Department of Computer Science and Automation, Indian Institute of Science, Bangalore, in 1988.

$\mathrm{He}$ is currently a Professor with the Department of Computer Science and Automation, Indian Institute of Science, Bangalore. He has consulted for several companies including Intel, General Motors Research, WIPRO, HCL, Satyam, and Tektronix. $\mathrm{He}$ is currently authoring a textbook on game theory and mechanism design. He has earlier coauthored a widely acclaimed textbook on Performance Modeling of Automated Manufacturing Systems (Englewood Cliffs, NJ: Prentice Hall, 1992). He has spent sabbaticals at the Massachusetts Institute of Technology, Cambridge, MA, in 1992 and at the National Institute of Standards and Technology, Gaithersburg, MD, in 1997. His current research focuses on the use of game theoretic and optimization models in computer science in general and electronic commerce in particular.

Dr. Narahari received the following awards: the IISc Best Ph.D. Thesis Award in 1988; Indo-U.S. Science and Technology Fellowship in 1992; the Sir C. V. Raman Young Scientist Award for Computer Science Research in 1998; Fellowship of the Indian National Academy of Engineering; and the Homi Bhabha Fellowship. He is currently on the Editorial Board of the IEEE TRANSACTIONS ON SYSTEMS, MAN AND CYBERNETICS (PART A) and the IEEE TRANSACTIONS ON AUTOMATION SCIENCE AND ENGINEERING. 